\title{
Célestin Lainé et le breton : la langue pour le combat
}

Célestin Lainé and Breton: a language for combat

\section{Sébastien Carney}

\section{(2) OpenEdition}

\section{Journals}

Édition électronique

URL : https://journals.openedition.org//bl/2241

DOI : $10.4000 / \mid \mathrm{bl} .2241$

ISSN : 2727-9383

\section{Éditeur}

Université de Bretagne Occidentale - UBO

\section{Édition imprimée}

Date de publication : 1 novembre 2011

Pagination : 151-197

ISBN : 978-2-901737-92-6

ISSN : 1270-2412

\section{Référence électronique}

Sébastien Carney, «Célestin Lainé et le breton : la langue pour le combat », La Bretagne Linguistique [En ligne], 16 | 2011, mis en ligne le 01 mai 2021, consulté le 22 mai 2021. URL : http://

journals.openedition.org/lbl/2241; DOI : https://doi.org/10.4000/lbl.2241

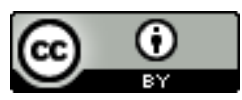

La Bretagne Linguistique est mise à disposition selon les termes de la Licence Creative Commons Attribution 4.0 International. 


\title{
Célestin Lainé et le breton : la langue pour le combat
}

\begin{abstract}
C
élestin Lainé et le breton : l'association ne coule pas de source pour qui connaît un peu le personnage. De ce militant nationaliste breton on sait surtout qu'il fit en 1932 exploser à Rennes le monument symbolisant le rattachement de la Bretagne à la France; et qu'il leva dès avant la Seconde Guerre mondiale une petite armée bretonne, plus tard baptisée «Bezen Perrot», après la mort de l'abbé du même nom en 1943. Cette armée participa activement aux côtés des troupes allemandes à plusieurs opérations meurtrières contre la Résistance, ce qui valut à Lainé ainsi qu'à certains de ses hommes d'être condamnés à mort par contumace pour intelligence avec l'ennemi en 1946. Dans la précipitation de l'action, la fureur des explosions, des coups de feu et des cris, on entend mal le breton s'exprimer, si ce n'est - l'anecdote a été maintes fois rebattue - à l'étonnement des Résistants et populations civiles bretonnes entendant ceux qu'ils prenaient à leur uniforme pour des «soldats allemands» parler le breton entre eux ${ }^{1}$.

* Doctorant en histoire, CRBC, UBO/ueb

1. Kristian Hamon, Le Bezen Perrot. 1944, des nationalistes bretons sous l'uniforme allemand, Fouesnant, Yoran Embanner, 2004, p. 101. Françoise MoRvan, Miliciens contre maquisards. Enquête sur un épisode de la Résistance en CentreBretagne, Rennes, Éditions Ouest-France, 2010, p. 65, 131, 133, 166.
\end{abstract}


Lainé a écrit, dans diverses publications, une centaine d'articles, dont un quart en breton, ou français et breton. On ne peut pas appeler cela une œuvre, or ceux qui l'ont connu savaient que Lainé écrivait énormément, au point que Per Denez demande en 1999 à Alan Heussaff, ancien du Bezen, si l'on peut espérer trouver quelque pièce d'intérêt dans les archives de l'ancien chef $^{2}$. En vain.

L'arrivée récente au CRBC d'archives personnelles de Célestin Lainé, conservées et léguées au Centre par Louis Feutren, un de ses anciens soldats exilé avec lui en Irlande, permet d'envisager le personnage sous un angle différent ${ }^{3}$. Ces archives ont été produites entre la fin de la Seconde Guerre mondiale et 1983, année de la mort de Lainé, entre-temps naturalisé irlandais sous le nom de Neven Henaff. Une exploration, toujours en cours, de ce fonds important permet de dresser un premier constat : les rapports entre Lainé et la langue bretonne ne sont pas anecdotiques. Celui qui se voulait prêtre et soldat d'une nouvelle foi celtique - druide en un mot - a peu publié, mais abondamment écrit, théorisé, en français, en breton, mais aussi en anglais, et en allemand à l'occasion. La langue bretonne a été au cœur de son combat, mais il s'agit moins, comme ce fut le cas pour d'autres militants de sa génération, d'un combat pour la langue, que d'une langue pour le combat. C'est ce que je vais exposer ici.

$*$

Célestin Lainé est né le 25 octobre 1908, quai de la Fosse, à Nantes ${ }^{4}$. Son père, originaire de Saint-Lunaire, en Haute-Bretagne, est marin au commerce, souvent embarqué. La famille s'installe bientôt à Brest, ce qui permet à sa mère native de Ploudalmézeau de se rapprocher de ses parents. Évoluant dans le milieu petit bourgeois de la Place du Château qui fréquente l'école Jean-Macé, se promène sur le Cours d'Ajot, va à l'église Saint-Louis et lit la Dépêche de

2. Institut de Documentation Bretonne et Européenne, Fonds Fouéré, Carton «Archives de Alan Heussaff»- lettre de Per Denez à Alan Heussaff, 14 janvier 1999.

3. Sébastien CARNEY, «Fonds Célestin Lainé», Le CRBC. 40 ans au service de la recherche sur le Bretagne et les pays celtiques, Brest, CRBC-UBO, 2009, p. 3537.

4. Sauf exception mentionnée, les informations relatives à l'enfance de Célestin Lainé viennent de CRBC, Fonds Lainé, CL1 T2 - autobiographie 1946. 
Brest, Lainé dit avoir reçu une éducation rigoureuse. C'est un élève brillant, "français et catholique toujours», évidemment antiallemand pendant la Grande Guerre et quelques années encore après 1918. Sa découverte de la langue bretonne, que sa mère connaît mais ne parle pas à la maison, a lieu pendant les vacances, qu'il passe à Ploudalmézeau ou à Portsall, chez ses grands-parents et ses cousins. À vrai dire, il découvre à la fois la langue et le milieu dans lequel elle est parlée. Et pour cet enfant de la III ${ }^{e}$ République, l'une ne va pas sans l'autre, et l'expérience est un peu douloureuse. Célestin, qui est déjà un enfant malingre, timide, manquant de confiance en lui, ne peut communiquer avec personne, même pas les membres de sa famille. «Le lundi, écrit-il, qui est le jour de foire et de marché à Ploudalmézeau, ce langage intrigant envahissait si totalement la maison et la famille que je me sentais isolé et réduit à la seule société de mes frères ». Sa mère, une fille Kerjean, parle elle-même ce «langage incompréhensible» avec les autres. Lainé trouve alors refuge auprès de son grand-père. Ancien marin, laïcard intransigeant, ce grand-père est aussi mécréant qu'ennemi du breton, bien que luimême bretonnant. Le breton est la "forteresse des gens d'Église» répète-t-il au petit Célestin ${ }^{5}$. C'est aussi la langue des ignorants, des ploucs et des cochons : il défend donc qu'on parle breton à ses petitsenfants de la ville, et à ceux-ci il interdit de jouer avec les autres enfants du village. Le grand-père n'a appris le français que vers ses 12 ans, et a regretté toute sa vie de ne pas bien le connaître. De fait, Lainé remarque bien le fossé qui le sépare de ses cousins de la côte dont la pauvreté le frappe. Aussi, l'isolement de Lainé n'est plus seulement linguistique, mais social :

«Mes frères et moi, ne parlant pas le breton, ne pouvaient pratiquement parler à personne de la famille pauvre, la quasitotalité. C'était senti comme très bien. Nous étions de l'Olympe silencieux et destinés, tout le monde le savait, aux plus grands honneurs. Et tout le monde s'en réjouissait et y eut concouru, car c'eût été une porte ouverte pour eux. Et de plus, pour vousmême, la conscience que vous ne réussissez rien "pour vous" qui ne soit aussi "pour la famille" - somme toute une vive conscience collective. C'était un succès pour la famille. On nous priait,

5. CRBC, Fonds Lainé, CL1 M12 - fragments autobiographiques, 1953. 
comme on prie en latin, on ne nous parlait pas, et nous ne pouvions répondre non plus que les dieux. Moi, j'en ai souffert - de cette apothéose familiale ${ }^{6} . »$

Le monde du petit Célestin se divise donc en trois catégories de Français : «les Français ignorants parlaient le breton et s'habillaient de même. Ils étaient la majorité à Ploudalmézeau et la minorité à Brest; c'étaient les paysans, les pêcheurs, les bonnes et les domestiques», puis viennent, dans la hiérarchie, les bilingues, et enfin «les français supérieurs ", ceux qu'on avait empêché d'apprendre le breton. Sa famille elle-même a intégré ce clivage social lié à la langue, et Lainé le comprend très bien :

«J'étais peiné de ne pouvoir guère communiquer avec eux, car mon breton les effrayait encore plus que mon français. Non pas qu'ils ne le comprissent pas, mais parce que cela, dans leur idée, offensait ma majesté. C'était quelque chose de monstrueux et d'abaissant, comme un dieu qui se fait homme ${ }^{7}$.»

Cette vision bipolaire partagée par Lainé et ses cousins est conforme à celle que partage la Basse-Bretagne au début du $\mathrm{XX}^{\mathrm{e}}$ siècle, laquelle sépare d'un côté le breton, la campagne, la misère, et de l'autre le français, la ville, le progrès ${ }^{8}$. Lainé sait donc très tôt quel est le chemin de la déchéance, de même que le catéchisme lui a appris quel est celui de l'enfer. Pendant son adolescence, Célestin explore ces chemins vertigineux, sans jamais se laisser basculer.

En effet, dans les années 1920, Lainé vit une série d'événements qui le troublent énormément. Prenant le train pour la première fois, il rejoint avec sa mère, son père alors en escale à Bayonne. Au carré de l'Orconera, les familles du capitaine Lainé, de son second et de son mécanicien se retrouvent dans la bonne humeur. En guise de plaisanterie, le capitaine s'exclame que la Bretagne devrait être indépendante. Tout le monde de rire, sauf le très français Célestin, alors prix d'excellence pour sa valeur morale, et dont le patriotisme est ici égratigné.

6. CRBC, Fonds Lainé, CL1 M12 - fragments autobiographiques, 1953.

7. CRBC, Fonds Lainé, CL1 M12 - fragments autobiographiques, 1953.

8. Sur ce schéma diglossique, voir Ronan CALVEz, La radio en langue bretonne. Roparz Hemon et Pierre-Jakez Hélias, Rennes, PUR/CBRC, 2000, p. 31-32. 
L'été arrive, il découvre dans le grenier de ses tantes le dictionnaire Breton-Français de Troude, qui aurait appartenu à son arrière grand-père. Le fait qu'une langue inculte ait un dictionnaire ne laisse de le surprendre. C'est vraisemblablement le même été que la famille reprend le train, pour Rouen cette fois-ci. Au retour, passant la nuit dans la gare de Serquigny, on subit les vociférations d'un «éthylique endiablé», débraillé, sale, portant une casquette de marin et un sac de matelot, accompagné d'une petite fille d'à peine 10 ans. La scène fait grande impression sur le sage Célestin qui se souvient en détail de cet qui homme qui :

«...pérorait, gesticulait et ne cessa crier jusqu'à l'arrivée du train : "Je suis Breton, moi! Je suis de Saint-Malo! Breton et pas Français! Breton et pas Français!" Un employé de gare, candide bretonnant exilé, tentait en vain de converser avec lui en breton. L'homme lui tapait sur l'épaule: "Oui mon pote! Tu es breton, toi aussi? C'est bien, ça! Moi je suis de Saint-Malo! I don't speak brezonek, mais je suis Breton quand même, Breton et pas Français ${ }^{9}$ !"»

Dans ses mémoires, se qualifiant d'«enfant de chœur bien élevé recueillant pieusement l'évangile de l'ivrogne», Lainé écrit que c'est à ce moment-là qu'il s'est lui aussi senti «Breton et pas Français». Cela ressemble à une invention, si ce n'était le dialogue, trop beau pour être faux, et on imagine sans peine l'impact de la scène sur un jeune adolescent en plein questionnement identitaire. Ce marin de Saint-Malo n'est pas sans rappeler son capitaine de père, mais plus loin encore, l'apparition nocturne de cette loque marine renvoie à un vieux fantôme familial, un autre Célestin, le grand-père Lainé, disparu en mer quand son fils avait l'âge de la fillette.

Dans le même temps, Lainé, alors en proie à une crise mystique importante, réfléchit à une vocation ecclésiastique, qu'il rejette finalement. Cette rupture progressive avec la foi chrétienne - au profit de son identité bretonne - semble avoir été aussi longue que difficile ${ }^{10}$. En 1922, Lainé semble se chercher, il est perméable à

9. CRBC, Fonds Lainé, CL1 T2 - autobiographie 1946, p. 9.

10. CRBC, Fonds Lainé, CL3 C171 - lettres d'Albert Lainé à Louis Feutren, 20 février 1984. 
toute sortes de signes nouveaux, et à certaines lectures, aux livres scientifiques, aux romans d'aventure. L'un d'entre eux, L'île du Solitaire $^{11}$, raconte l'histoire d'un scientifique qui ambitionne d'imposer sa volonté au monde. Le jeune lecteur, pour qui la Bretagne semble être un véritable projet, imagine de son côté ériger un État breton à l'aide d'une armée bretonne devenue surpuissante grâce à la science. Désormais, Célestin se consacre donc à ses études, et à l'apprentissage du breton.

En octobre 1923, sa professeur d'espagnol demande à la classe qui connaît le breton : à peine un quart le parle, ce qui le chagrine, d'autant que l'enseignante apprécie que l'on parle cette langue. Il se demande alors comment apprendre le breton, fait part de son désir à sa mère, laquelle lui donne 5 francs pour aller à la librairie Derrien, rue de Siam, acheter un livre. L'achat prend l'allure d'une aventure magique et mystérieuse :

«Il commençait à faire sombre. Je bondis jusqu'à la librairie Derrien avec la crainte qu'ils n'aient déjà fermé. Mais pas encore ! Tout soufflant, j'exposai ma requête à la vieille dame. Mais oui, elle avait cela! D'un recoin obscur et poussiéreux elle extirpa non seulement un, mais deux, mais trois, mais beaucoup de bouquins de différents types. Je choisis un exemplaire du Vallée et comme il ne coûtait que trois francs cinquante, je m'enhardis à acquérir, en sus des instructions maternelles, un vénérable Divizou Gallek ha Brezonek qui ne coûtait alors que dix sous...

Je rentrai triomphant avec mon butin ${ }^{12} . »$

Butin qu'il abandonne provisoirement devant la charge de travail que nécessite le baccalauréat, qu'il obtient avec la mention «assez bien». Il met l'été et ses vacances à Ploudalmézeau à profit pour engranger du vocabulaire breton, puis à la rentrée, valide l'examen d'entrée en classe de Mathématiques élémentaires, tout en s'inscrivant au cours de Philosophie. C'est en novembre 1924 qu'il entend parler d'une revue publiée par une ligue de Rennes, il s'agit de Breiz Atao. L'achat de son premier exemplaire de la revue prend la tournure d'un récit initiatique :

11. Maurice Champagne, L'île du solitaire, Paris, Librairie Delagrave, 1924.

12. CRBC, Fonds Lainé, CL1 T2 - autobiographie 1946, p. 12. 
«La librairie Robert était une minuscule boutique attenant ou presque à la Brasserie de la Marine. Jamais auparavant je ne l'avais remarquée. Tiens, tiens! Un carton à la vitre: "On trouve de tout en Bretagne, même des Bretons. On les reconnaît à ce qu'ils lisent Breiz Atao". Aucun doute, c'est là. J'entre le cœur battant et je fais ma demande de mon ton le plus naturel qui dans la circonstance, était aussi le plus artificiel. Le petit vieux me répond affirmativement, atteint un numéro, le roule en toute quiétude et l'enveloppe d'un papier blanc. Me voici à la rue, mon rouleau à la main. Je ne vois plus ni le ciel ni la terre mais seulement ce rouleau blanc qui me possède des pieds à la tête. Jamais aucune fée n'a senti autant de pouvoir dans la baguette qu'elle tenait à la main. Tant de pouvoir et aussi tant d'anxiété : ce qui est écrit là-dedans, est-ce bien ce que je veux? Ces gens de Rennes où l'on ne parle plus le breton, ces gens de Rennes qui veulent une Bretagne libre, entendent-ils bien par là une Bretagne faisant bonne place à la langue bretonne? Je veux être fixé tout de suite mais je ne puis tout de même pas déflorer le rouleau dans la rue, devant ces passants étrangers. Non, non! À la maison, seul! Pas de spectateurs contraints de mes triomphes ou désillusions. (...) J'entends mes sabots qui claquent sur les dalles de granit. Je courais donc depuis un instant déjà? Je me lance dans la rue du Château. J'ai des ailes. En franchissant la rue Traverse je m'entends dire : "Mon Dieu! Si c'est ce que je pense, alors c'est toute ma vie que je tiens là entre mes mains..." Ai-je parlé ou a-t-on parlé? Je ne sais. Mais maintenant c'est moi $^{13}$ qui réponds avec ferveur : "Oui, c'est vrai ! C'est toute ma vie qui est là entre mes mains!" Je vole dans les airs. D'une haleine je franchis les quatre étages qui conduisent à la chambre-mansarde que je partage avec mon frère Francis. Je m'installe dans ma fenêtre à moi. La mer, Plougastell et l'île Ronde, le Menez Hom, mon pays est là luisant et dégagé, devant moi, avec moi. Il est la seule compagnie que je tolère à une heure aussi solennelle. Tremblant et fiévreux, je déploie respectueusement le rouleau en murmurant des prières machinales. Voilà la couverture, noir et vert sur fond blanc ${ }^{14}$. Je la tourne. Mon cœur saute dans ma poitrine... ça y est ! Ça y est : du breton dès la première page! Rien à craindre désormais! Je

13. C'est Lainé qui souligne.

14. C'est le $\mathrm{n}^{\circ} 12(72)$ du $1^{\text {er }}$ décembre 1924 dont la couverture est décrite ici. Par contre, c'est dans le numéro précédent que l'on trouve du breton dès la première page. 
n'essaie pas de le lire, ce serait trop long. Je saute aux articles en français... C'est cela! Exactement cela. D'un bout à l'autre ! Dieu soit loué!

Le tonnerre eut pu tomber que je n'en aurai fait aucun cas. Mais il y eut plus fort que le tonnerre : un ordre maternel bref et comminatoire m'enjoignit de descendre pour manger et m'arracher à ma lecture. Toutefois il ne put m'empêcher qu'avant le soir je ne l'eusse bien réitérée cinq ou six fois.

Voilà comment, à l'âge de seize ans révolus, je fis la connaissance de Breiz Atao $^{15}$.»

On est ici en terrain désormais familier : c'est encore dans un petit endroit mystérieux qu'une personne âgée délivre l'ouvrage - la «baguette»-magique. La nouveauté réside dans l'aspect sexué de la révélation. Ce qu'on en retiendra c'est le parallèle entre Breiz Atao, la langue bretonne, et sa virilité que Célestin découvre. C'est ce que confirme le caractère guerrier de son adhésion à l'Unvaniez Yaouankiz Vreiz, qu'il rejoint «comme un volontaire qui s'engage pour la durée de la guerre (...) résolu à lui offrir sans barguigner au moins une ou deux pintes de (son) sang ${ }^{16} \gg$. Désormais, il reçoit Breiz Atao au grand dam de ses parents, et travaille le Vallée une demi-heure par soir, avant de s'endormir. Les gens de l'U.Y.V. le dirigent vers Youen Drezen, alors rédacteur au Courrier du Finistère. C'est avec plaisir qu'il fréquente Drezen, qui lui conseille l'achat d'Emgann Kergidu, du Barzaz Breiz, et de l'Histoire de notre Bretagne, de $\mathrm{M}^{\mathrm{me}}$ du Guerny, laquelle attise fortement sa francophobie. Drezen le présente à Roparz Hemon, alors jeune professeur d'anglais. Hemon lui offre $L a$ vie de Salaün de Tanguy Malmanche, en français. L'ouvrage lui fait très grande impression. Il rencontre enfin Jakez Riou. Avec eux il commente les articles de Breiz Atao, évoque les combats de 1'U.Y.V., mais les trois compères échangent en breton, tout en s'adressant en français à Lainé, lequel en est très vexé ${ }^{17}$.

Au mois de juillet 1925, il se rend à Rennes passer ses examens : il obtient la mention bien à Mathélem, assez bien en Philosophie. Il a par contre moins de succès lors de ses visites rue du Vau Saint-

15. CRBC, Fonds Lainé, CL1 T2 - autobiographie 1946, p. 14-15.

16. Id., p. 15 .

17. CRBC, Fonds Lainé, CL1 M1 - autobiographie 1941. 
Germain, où est domicilée l'U.Y.V., chez Debauvais, toujours absent. Il a aussi moins de succès à la maison où il se sent devenir étranger à la famille. Les disputes se multiplient avec sa mère qui s'inquiète de l'avenir de son fils influencé par ses lectures bretonnes choquantes. En 1926 il obtient malgré tout le prix d'excellence de la classe de Flotte et est admis commissaire dans la promotion 1926 de l'École Navale. Mais la fièvre typhoïde met fin à sa carrière maritime, et tue sa mère, en septembre 1926.

En février 1927 il s'inscrit à la faculté des sciences de Rennes. Là il suit les cours de celtique peu fréquentés de Le Roux à la faculté des Lettres ${ }^{18}$, cherche à se faire des amis au Cercle SaintYves des étudiants catholiques, rencontre Morvan Marchal auprès duquel il éprouve ses convictions religieuses. L'U.Y.V. le déçoit : les glorieux combats qu'il avait imaginés se résument à verser une petite cotisation, vendre un journal, porter un insigne et assister aux réunions d'une section fantomatique. Aux vacances 1927, il rencontre l'abbé Perrot par l'entremise d'un ami du cercle Saint-Yves, originaire de Plouguerneau. C'est assidûment qu'il fréquente l'abbé, en qui, dans sa solitude, il trouve un père. Le même été a lieu le Congrès de Rosporden où est fondé le Parti Autonomiste Breton. Lainé s'y rend avec Hemon, rencontre Yann Sohier avec lequel il peint des drapeaux - le Gwenn ha Du de Marchal - et adhère au tout nouveau parti. Lainé entre au P.A.B. comme on entre en religion. Le nouveau membre de la communauté se plie à une règle - des statuts -, se réunit à intervalles réguliers avec ses frères - les réunions hebdomadaires de la section - et enfin change de nom. Bretonnisant son état-civil, il devient Gwenael An Henaff. Le procédé est tellement courant dans le mouvement breton ${ }^{19}$ qu'on n'y prêterait guère attention. Cependant, dans ce cas précis où il ne s'agit pas d'accumuler les signatures dans quelque publication, c'est bel et bien une démarche à caractère religieux à laquelle se livre Lainé. L'exhortation au sacrifice prônée par le parti passe, comme au monastère, par le reniement de soi, de

18. Pierre Le Roux est connu pour avoir publié, entre autres, un Atlas linguistique de la Basse-Bretagne, Rennes-Paris, Plihon et Hommay, Honoré Champion, 1924. Voir François FalC'Hun, Nécrologie : Pierre Le Roux, s.n., 1976.

19. Jean Malo Renault, Les pseudonymes des Bretons, $16^{e}-20$ e siècle, Volume I, Brest, Ar Skol Vrezoneg, Emgleo Breiz, 1994, p. 16. 
son identité propre, au profit d'une autre, communautaire ${ }^{20}$. Et pour Lainé, la conversion n'est pas vraiment simple. De son propre aveu, le nom de Gwenael ne lui convient pas ${ }^{21}$, d'ailleurs il signe plutôt $G$. ou Gw. Son entourage l'appelle Nael, un surnom dont Olier Mordrel revendique la paternité ${ }^{22}$. Il changera encore de prénom quelques années plus tard.

Au printemps 1928, il valide avec succès sa licence de mathématiques. Dans le même temps, sa connaissance du breton a bien progressé grâce à un ami qui lui parle exclusivement en breton et exige qu'il lui réponde de même, à sa fréquentation des cours de celtique où ses copies font bonne impression, et à sa lecture assidue des récits épiques irlandais publiés dans Gwalarn. Roparz Hemon lui parle alors du Simbol ${ }^{23}$, auquel Lainé s'inscrit et est admis par ses amis Hemon, Riou et Drezen ${ }^{24}$. C'est une grande fierté pour lui de porter uniquement l'insigne du Simbol, qu'il préfère à l'hévoud ${ }^{25} \mathrm{du}$ P.A.B., bien plus facile à obtenir. Le même été il éprouve son breton avec Perrot et une équipe de pèlerins plouguernéens, sur la route de Sainte-Anne d'Auray. Arrive la rentrée, la fin des pèlerinages, des congrès et autres fêtes celtiques, Célestin part à Paris préparer un diplôme de chimie générale à la Sorbonne : il est reçu avec la mention «assez bien». Là, il chante en breton au cercle celtique de Mr Régnier, suit les cours hebdomadaires de l'abbé Léon, à la Sorbonne. Il participe à la fondation d'une section de la Fédération des Étudiants Bretons dont il devient secrétaire. Avec ses camarades il rédige une affiche bilingue, collée illégalement de nuit et aussitôt recouverte par d'autres, timbrées celles-là. Il aurait également dessiné la célèbre carte postale de propagande intitulée "Ablamour m'en deus komzet brezoneg ${ }^{26}{ } »$. Les réunions de la section le déçoivent :

20. Danielle Rives, «Mourir au monde et renaître au divin : le nom en religion», dans Agnès Fine (dir.), États civils en questions. Papiers, identités, sentiment de soi, Paris, Édition du CTHS, 2008, p. 179-201.

21. CRBC, Fonds Lainé - CL1 M15.

22. Olier Mordrel, Breiz Atao. Histoire et actualité du nationalisme breton, Paris, Alain Moreau, 1973, p. 204.

23. Association créée par Roparz Hemon, s'attachant à l'enseignement du breton.

24. Voir Gwalarn, n 19, gouere 1929, p. 102.

25. Croix gammée, insigne du parti.

26. En français, «Parce qu'il a parlé breton». Ce dessin est publié le 7 octobre 1928 dans Breiz Atao. Lainé en revendique la réalisation (CL3 C163 - brouillon 
un Duhamel, craignant la mauvaise publicité que cela pourrait lui faire, aurait refusé de participer à une conférence publique. Lainé se concentre alors sur ses études et ses cours de breton. En 1929 il entre à Centrale, d'où il ressort ingénieur diplômé deux ans plus tard. À la Sorbonne, il remplace quelques mois l'abbé Léon dans ses cours. En été 1931, il est examinateur pour le Simbol aux assises linguistiques de la première école d'été de Saint-Goazec.

Ces dix années de la vie de Lainé permettent de constater plusieurs choses. D'une part on l'a vu rompre avec les ambitions familiales francophiles, avec ses parents, au point que, devenu vieux, il se sentira responsable, par son engagement breton, de la mort de sa mère ${ }^{27}$. Ses convictions religieuses commencent à s'effriter peu à peu. Sa vie de militant se résume à de multiples déceptions. Ses succès sont ailleurs. Il est intéressant de constater comment Lainé clôt simultanément ses études, ainsi que son apprentissage du breton : il devient ingénieur en même temps que professeur, au point d'être bientôt considéré comme un excellent bretonnant ${ }^{28}$. C'est assez logiquement que ces deux domaines - science et Bretagne, ou plutôt science et breton - se rejoignent, dès 1932, dans l'action de Gwenn ha Du, et la rédaction de Mentoniez.

$*$

Dès la fin des années 20, Lainé entame la rédaction d'un ouvrage de géométrie, dont il livre les premiers traits dans Gwalarn ${ }^{29}$. Ce faisant, il rejoint avec son collègue Berthou - lequel s'intéresse à l'électricité et la chimie ${ }^{30}$ - une élite de jeunes bretonnants célébrée

de lettre de Célestin Lainé à Caouissin, février 1971), alors qu'Olier Mordrel l'attribue à Yann Sohier (O. Mordrel, Breiz Atao. Histoire et actualité du mouvement breton, op. cit., p. 118), ce dont doute fortement la fille de ce dernier.

27. CRBC, Fonds Lainé, CL1 M16 - fragments autobiographiques 1963, «Tokyo, April 291963 - souvenirs» et CL1 M28 - fragments autobiographiques.

28. IDBE, archives Fouéré, Carton A.B.E.S., Organisation intérieure, action extérieure, actions parlementaires, professions de foi (législatives), 1935. Lettre de Robert Audic à Yann Fouéré, 28 février 1937.

29. H..., «Ur pennad mentoniez», Gwalarn, $n^{\circ}$ 17, printemps 1929, p. 87-91 (attribué à Lainé dans l'index du n ${ }^{\circ} 131$ ).

30. Gw. Bertou, «Eur pennad strilhouriez», Gwalarn, n 17, printemps 1929, p. 87-91 ; et Gwilherm BerThou, «Tredan ha Gwagennou», Gwalarn, n²0, 1930 , p. $77-87$. 
par Debauvais, qui déplore toutefois son faible effectif ${ }^{31}$. La rédaction de l'ouvrage est achevée alors qu'il travaille comme chimiste à Loos, dans le Pas-de-Calais, entre 1932 et $1934^{32}$. À la fin de l'année 1934, Gwalarn annonce «une grande nouveauté. Le premier d'une série de livres savants en breton ${ }^{33} »$. La parution de Mentoniez ${ }^{34}$ est saluée comme un événement historique.

«Mar deo gwir e pouez an darvoudou speredel muioc'h eget an darvoudou politikel e buhez eur bobl, eun darvoud bras zo en em c'hoarveset en istor Breiz e miz du 1934. Er miz-se eo bet embannet eul levr hag a ro kement a lañs hag a sked d'hor yez vroadel ma kredan e vo eñvoret da viken gant hor gourbivien, evel Geriaduriou Ar Gonideg ha "Barzaz Breiz” Kermarker. (...)

Diaes e vo d'ar re n'o deus ket c'hoaz al levr kompren al levenez hag al lorc'h a zo ennoun pa gomzan anezañ ${ }^{35}$.»

(S'il est vrai que les événements spirituels pèsent davantage dans la vie d'un peuple que les événements politiques, il s'est produit en Bretagne un grand événement en novembre 1934. Ce mois-là a été publié un livre qui donne tant d'élan et d'éclat à notre langue nationale que je crois que nos militants s'en rappelleront à jamais, à l'instar des Dictionnaires de Gonidec et du «Barzaz Breiz» de La Villemarqué. (...)

Ceux qui ne possèdent pas encore le livre auront du mal à comprendre la joie et la fierté qui sont les miennes quand j'en parle.)

Lainé est félicité par un instituteur ${ }^{36}$. «On peut même dire que le vocabulaire de la géométrie est actuellement plus scientifique en breton qu'en français », écrit François Kervella, paraphrasant Lainé lui-même ${ }^{37}$. Un autre lecteur prétend même que l'acquisition de la

31. F. Debauvais, «Hag ar brezoneg ?», Breiz Atao, n 87, 8 février 1930, p. 1.

32. CRBC, Fonds Lainé, CL1 M1 - autobiographie 1941.

33. Non signé, publicité, Gwalarn, n 71, octobre 1934, p. 64. «Eun nevesenti vras. An hini kenta eus eur rummad levriou gouiziegez e brezoneg». Une cinquantaine d'ouvrages était prévue.

34. C.L. KerJeAn, Mentoniez, Brest, Gwalarn, 1934.

35. Non signé, «Mentoniez», Gwalarn, n 73, décembre 1934, p. 55-56.

36. Non signé, "Atao diwar-benn ar "Ventoniez"», Gwalarn, n 77, avril 1935, p. 54.

37. D.K., «Les livres. Mentoniez», Breiz Atao, n² 212, 2 décembre 1934, p. 3, et C.L. KerJeAn, Mentoniez, Brest, Gwalarn, 1934, «Adskrid ha Geriadur», p. 1. 
géométrie lui est plus facile et plus distincte en breton : qu'attend-on pour diffuser ce livre dans les écoles, demande-t-il ${ }^{38}$ ?

Effectivement, le livre est assez bien conçu. Même si la maquette est austère, et le contenu assez aride, on ne peut que remarquer le souci pédagogique affiché par l'ouvrage, lequel, vraisemblablement inspiré de ceux que Lainé a utilisés dans son propre cursus, est clairement découpé en chapitres thématiques, compte de nombreuses figures numérotées et expliquées, des définitions, des exercices, et enfin un livret de vocabulaire dépliable de façon à être consulté en regard du texte. On remarque aussi que c'est du nom de Kerjean, celui de sa mère et de ses grands-parents, qui lui ont payé ses études à Centrale, que Lainé, se désignant comme scientifique et breton dans l'avantpropos du lexique ${ }^{39}$, signe Mentoniez. Faut-il voir une tentative de réconcilier les ambitions sociales de la famille avec son intérêt pour la cause bretonne? Le mouvement - se souciant davantage de son propre avenir que du passé de ses militants - ne se pose pas ce genre de questions. Roparz Hemon explique :

«Kement-se a dle lakaat lorc'h er vrezonegerien. Gwaz a se da dud ar rummad koz ha n'int ket evit kompren talvoudegez seurt labouriou, ha d'ar c'hallegerien a chom sabatuet ganto. N'eo ket evit ar re-se e labouromp. Evit ar re yaouank eo, evit Breiz a vo, ha nann evit Breiz a zo na Breiz a zo bet. Ne reomp forz eus ar rebechou (nemet graet e vefent gant tud doujus, gouiziek hag ampart). Touet hon eus adsevel hor yezh hag hor bro, ha kemer a reomp an hent eeun evit hen ober ${ }^{40}$. »

(Une telle chose doit remplir les bretonnants de fierté. Tant pis pour les gens de la vieille génération qui ne peuvent comprendre la signification de ces travaux, et pour les francisants qui en restent stupéfaits. Ce n'est pas pour eux que nous travaillons. C'est pour les jeunes, pour la Bretagne à venir, et non pour la Bretagne actuelle ni la Bretagne passée. Nous ne nous soucions guère des reproches (à moins qu'ils ne soient le fait de person-

38. Non signé, «Ar “Ventoniez"», Gwalarn, $\mathrm{n}^{\circ}$ 75, mars 1935, p. 49-50 : «Aesoc'h, ha fraesoc'h eget e galleg e teu traou ar ventoniez e brezoneg. Petra a c'hortozer da lakaat al levr-se er skoliou?».

39. C.L. KerJean, Mentoniez, Brest, Gwalarn, 1934, «Adskrid ha Geriadur», p. 1. 40. Non signé, «Ar “Ventoniez"», Gwalarn, n 75, mars 1935, p. 49-50. 
nes respectueuses, savantes et compétentes). Nous avons juré de relever notre langue et notre pays, et nous empruntons la route directe pour y parvenir.)

Malgré les ambitions pédagogiques, la question est bel et bien de savoir à qui s'adresse un tel livre : «Pourquoi un tel manuel pour quelques dizaines de lecteurs qui seront capables d'en tirer profit?» demande Kervella :

«C'est qu'avant tout ce livre est un acte de foi en l'avenir de la langue bretonne. Peu importe qu'il n'y ait pour le moment que quelques personnes à s'intéresser à la géométrie en breton. On ne vise pas ici à l'étude de cette science, on vise à montrer que le breton pourra servir, le moment venu à enseigner toutes branches du savoir humain ${ }^{41} . »$

L'affaire est claire, Mentoniez n'a pas été écrit pour être lu, ni utilisé, ni même compris : il s'agit d'un "acte de foi en l'avenir de la langue bretonne». Cet acte de foi passe par l'élaboration d'un vocabulaire nouveau, d'une liturgie scientifique nouvelle en langue bretonne. Lainé, conscient d'avoir été confronté à un vide lexical, se défend d'avoir recours à des «créations erronées de l'impétuosité des érudits ${ }^{42}$ », mais bel et bien à des mots véritablement populaires, répondant à de véritables besoins. Ce vocabulaire nouveau a été validé par Roparz Hemon, lequel a testé l'authenticité raciale de chacun d'entre eux ${ }^{43}$. Il s'agit de proposer un breton dont les générations passées n'aient pas honte, et dont celles qui suivent puissent être fières : «un breton celtique». Aussi, à chaque fois que cela a été possible, Lainé s'est servi de mots anciens, communément utilisés. Dans cet esprit, cercle devient kelc'h et ligne devient linenn, ainsi que chaque bretonnant contemporain l'entend. Pour autant, le contenu reste difficilement compréhensible, comme le fait remarquer Yves Le Moal, dans son journal Breiz : «... digoromp al leor, forz e pe bajenn : p76 : Adwirienn. - Kreizserzennou tuiou pep tric'horn a

41. D.K., «Les livres. Mentoniez», Breiz Atao, n² 212, 2 décembre 1934, p. 3.

42. «krouaduriou faltazi diroll ar Ouizieien», C.L. KerJeAn, Mentoniez, Brest, Gwalarn, 1934, «Adskrid ha Geriadur», p. 1.

43. «...n'eus ket unan eus ar geriou nevez-mañ a vefe bet embannet hep beza bet digantañ eun destenn a ouenn vat», C.L. KERJEAN, op. cit., p. 1. 
gej en eur poent a zo kreizenn ar c'helc'h a dremen dre e dri beg. (Corollaire : les perpendiculaires au milieu des côtés d'un triangle se rencontrent en un point qui est le centre du cercle qui passe par les trois angles) ${ }^{44}$.» Prétendu populaire, le breton défendu par Lainé dans son ouvrage, doit surtout être une langue fidèle au supposé génie de la race. Ainsi, quelques mois après la parution de Mentoniez, Lainé propose dans Gwalarn quelques modifications de vocabulaire, apportées après concertation avec Hemon, François Vallée et Raymond Delaporte. Les mots d'origine trop commune, latine ou grecque, en un mot française, on été remplacés par des mots plus précis, celtiques. Boule n'est plus boull, mais pellenn, par exemple ${ }^{45}$. Ce faisant, Lainé s'inscrit clairement, et sans surprise, dans l'école gwalarnienne, telle qu'elle s'est définie dans son manifeste, en 1925 : «réaction de la jeunesse cultivée contre les modes surannées et la fausse paysannerie», laisser le soin à l'élite de créer une littérature bretonne qui s'efforce «de puiser sa sève dans le génie de la race ${ }^{46}$ ». À ce stade, on ne distingue pas forcément très bien ce qui est fait pour le breton de ce qui l'est contre le français.

On sait comment l'attentat de 1932 contre la statue de Boucher célébrant l'union de la Bretagne à la France permit de relancer une action bretonne engluée dans ses divisions et ses soucis financiers. On sait aussi comment la société secrète Gwenn ha $D u$, zone grise du drapeau blanc et noir, fit sensation à cette occasion. On oublie par contre, derrière les boîtes de Gloria piégées, les rails coupés et les articles dans Détective, que ce petit groupe d'activistes dirigés par Lainé communiquait aussi en breton, et plaçait la langue bretonne au cœur de ses revendications ${ }^{47}$. Ainsi, le $1^{\text {er }}$ mars 1936, Breiz Atao

44. «Ouvrons le livre à n'importe quelle page : p76...». E.A.M., «Mentoniez», Breiz, $n^{\circ} 384,2$ décembre 1934, p. 1. Ce passage n'est pas repris dans la recension de l'article d'Yves Le MoAL dans non signé, «Al levr "Mentoniez”», Gwalarn, $\mathrm{n}^{\circ} 74$, janvier 1935 , p. $54-55$.

45. C.L. KerJeAn, «Diwar-benn "Mentoniez” ha Yez ar Skiantou», Gwalarn, n 80, juillet 1935, p. 53-56.

46. Roparz Hemon et Olivier Mordrel, «Gwalarn», Breiz Atao, n 2 (74), $1^{\text {er }}$ février 1925, p. 524.

47. Indirectement cependant, l'attentat de Rennes contribua à la cause de l'enseignement du breton. Une chanson sur feuille volante, intitulée Kanaouenn war sujed torfed roazon (chanson sur le thème du crime de Rennes), fut publiée et vendue 
publie une lettre datée du 15 février dans laquelle Gwenn ha Du pose un ultimatum au gouvernement français quant à l'enseignement du breton :

«15 a viz C'houevrer 1936

65 vloaz 'zo, eman ar Vretoned o c'houlenn o yez da veza desket er skoliou. Abaoe daou vloaz, 120 Kuzul-kêr, 27 Kevredadgouizieien ha tri C'huzul-Meur Goueled-Breiz a-unvouez, o deus diskouezet sklaer o mennad dre vouezia a-du ganti.

E mis mae 1935, Gouarnamant Bro-C'hall en deus nac'het krenn rei e c'houlenn da Guzul-Meur ar Morbihan, ha d'ar 16 a viz kerzu 1935 en deus graet skouarn vouzar ous galvadenn daou gannad breton e Kambr ar Gannaded.

Ar SEIZVET gwech eo d'ar C'hallaoued, abaoe 20 vloaz, ober seurt dinac'h-gwir da Vreiz, ha teuret ken gwas dismegans warni.

N'hon eus fizians ebet ken er Gouarnamant da rei d'imp hor gwir dre gaer.

Dre heg e fell d'imp breman hel lakaat da anzav hor gwiriou.

Ne c'houzanvimp ket mui e talvezfe hor skoliou da zeski d'hor bugale kasaat o yez, ober fae war o zadou-koz ha kaout mez gant o gouenn.

Eur miz, adalek hizio, a askouezomp gant an Ao. GUERNUT, rener ar Gelennadurez, da lakaat kelenn ar brezoneg a-berz-stad e holl skoliou Goueled-Breiz.

\section{Dre urz : \\ GWENN HA DU»}

Traduction :

Voici 65 ans que les Bretons demandent l'enseignement de leur langue dans les écoles. Depuis deux ans, 120 Conseils Municipaux, 27 Sociétés Savantes et les trois Conseils Généraux de la Bretagne bretonnante à l'unanimité des voix, ont manifesté clairement par leurs votes leur volonté de l'obtenir.

Au mois de mai 1935, le Gouvernement français a refusé nettement de donner satisfaction à la demande du Conseil Général du Morbihan, et le 16 décembre 1935 il est resté sourd à l'intervention de deux députés bretons à la Chambre des Députés.

au profit d'Ober, association dirigée par Marc'harit Gourlaouen dispensant des cours de breton par correspondance. 
C'est la septième fois que les Français, depuis 20 ans, renouvellent ce déni de justice à la Bretagne et lui font cet inqualifiable affront.

Nous ne croyons plus que ce Gouvernement reconnaitra notre droit de bon gré.

Nous entendons désormais lui en imposer la reconnaissance par la force.

Nous ne supporterons plus que nos écoles servent à apprendre à nos enfants la haine de leur langue, le mépris de leurs ancêtres et la honte de leur race.

Nous accordons un mois, à dater d'aujourd'hui, à M. Guernut, Ministre de l'Éducation Nationale, pour organiser l'enseignement officiel du breton dans tous les établissements scolaires de la Bretagne bretonnante.

Par ordre :

BLANC-et-NOIR ${ }^{48}$.

Le communiqué fait ici clairement référence aux interventions de l'abbé Desgranges et des députés Trémintin et Inizan à l'Assemblée nationale en 1935, ainsi qu'à l'action d'Ar Brezoneg er Skol, alors toute jeune association créée par Yann Fouéré. Ce dernier, à force de courriers, de déclarations, de vœux, dépense une énergie considérable afin de tisser un réseau de personnalités favorables à l'enseignement du breton. Breiz Atao souligne d'ailleurs le danger de confusions possibles entre l'action des deux groupes, l'un clandestin et violent, l'autre légal et pacifique. L'action de Gwenn ha Du «...dérangerait la politique des promoteurs de Brezoneg er Skol et retarderait l'admission de la langue bretonne dans nos écoles», prévient Breiz Atao, qui cautionnera malgré tout les attentats consécutifs au non respect de l'ultimatum.

Deux ans plus tard, ce sont les tensions internationales, autour de la crise des Sudètes, qui conduisent Lainé et son équipe à écrire des slogans sur les murs de Bretagne, à l'exemple de Flor(imont) Grammens, activiste flamand populaire dans le mouvement breton dès $1938^{49}$. Grammens se fit une solide réputation de barbouilleur

48. Gwenn ha Du, «Lettre du 15 février 1936», Breiz Atao, n 244, $1^{\mathrm{er}}$ mars 1936 , p. 2. La traduction est proposée par Breiz Atao.

49. Anonyme, «Les exploits du barbouilleur Grammens», Peuples et Frontières, $\mathrm{n}^{\circ} 8,15$ janvier 1938, p. 201-202. 
de panneaux de signalisation, qu'il voulait voir rédigés en flamand. Or, contrairement à lui, l'action des barbouilleurs bretons ne se place pas sur le plan linguistique mais politique. Les slogans sont d'ailleurs pour la plupart rédigés en français : «La France est foutue», «Les Français dehors», "Contre la guerre, Bretagne libre et neutre», peuton lire un peu partout en Bretagne. «Breiz Atao», lit-on en breton ${ }^{50}$. Les barbouilleurs, dont Lainé, sont arrêtés et jugés. C'est au cours du procès, auquel Lainé aurait voulu l'y faire entendre, que le breton reparaît.

Le 22 juin 1938, les barbouilleurs bretons sont jugés à Rennes. Lainé fait sensation en refusant de s'exprimer autrement qu'en breton. Une déclaration, dans laquelle il endosse toute la responsabilité des barbouillages, doit être lue par son avocat :

« Je suis Breton et j'ai résolu de ne répondre que dans ma langue nationale pour attirer l'attention sur la manière dont notre langue bretonne est traitée en Bretagne, dans notre pays, du fait de la domination et des lois françaises. (...) L'instruction ayant refusé de m'entendre jusqu'ici, je me vois réduit à faire lire par mon avocat cette traduction française de la déclaration que j'aurais faite si les autorités françaises avaient consenti à entendre ma langue nationale. Il m'aurait été très honorable d'être jugé et condamné sans avoir même été entendu parce que les lois françaises ne veulent pas admettre l'existence officielle de notre langue bretonne; aussi est-ce uniquement la préoccupation du sort de mes jeunes camarades ici présents qui me décide à faire lire cette traduction française, ce dont je prie tous les Bretons de m'excuser ${ }^{51} \cdot »$

Le procédé n'est pas nouveau, là encore Lainé s'inspire du modèle d'activistes étrangers : en 1937, les nationalistes gallois Saunders Lewis, Lewis Valentine, et D.-J. Williams passent en jugement après avoir incendié une base anglaise. Pendant le procès, ils veulent parler gallois, on le leur refuse. Lainé connaît bien les faits, pour avoir lui-même couvert l'événement dans la revue Peuples et

50. Après le procès, les formules sont plus radicales : «Ar vretoned mestr en o bro», ou encore «la France aux juifs, la Bretagne aux bretons».

51. C. LAINÉ, «Déclaration de Lainé», Breiz Atao, n 305, 26 juin 1938, p. 1. 
Frontière $^{52}$. Leur exemple lui permet de devenir celui que Breiz Atao présente comme «le premier Breton qui refusa de parler français aux juges étrangers ${ }^{53} \gg$. Véritable icône de la presse nationaliste de la fin des années 30, Lainé ne cherche pourtant pas le vedettariat. Peut-être vise-t-il bien plus haut. «En me conduisant ainsi j'ai été le défenseur de tous les paysans et ouvriers bretons qui sont traités comme des inférieurs parce qu'ils ne connaissent que la langue qu'ils ont apprise dans les bras de leurs mères », écrit-il en septembre $1938^{54}$. Il y a peut-être dans ces lignes une pensée pour sa grand-mère Kerjean, décédée au début de l'année, et un véritable engagement pour la langue bretonne. Mais il y a là une prétention christique assez nette. Au sujet des nationalistes gallois, Lainé écrivait déjà :

«Les hommes qui sont assez braves pour risquer une situation considérable, leur liberté et leur vie, n'ont pas besoin d'autre approbation que celle de leur conscience, n'ont pas besoin d'autre juge que $\mathrm{Dieu}^{55}$.»

Son refus de s'exprimer autrement qu'en breton ferait alors de cette langue non pas le but mais l'outil d'un combat. Ce n'est pas pour mais par le breton que lutte Lainé. Par ailleurs, le breton est, en ce qui le concerne, le vecteur de ce qu'il souhaite être sa passion de soldat christique. C'est ce que tendent à prouver ses écrits des années 1930-1940.

Contrairement à l'image que l'on se fait du personnage, Lainé a aussi donné dans la poésie. Certains de ses poèmes ont même été publiés, sous le pseudonyme d'Urien Riwallon, dans Gwalarn. Lainé y fait part de sa tristesse consécutive à une rupture avec un ami, vraisemblablement en désaccord avec ses activités bretonnes ${ }^{56}$. Peut-être songe-t-il à sa mère, en proposant un voyage vers Tir $\mathrm{Na}$ $\mathrm{N}-\mathrm{og}$, le paradis des Celtes :

52. Allbrogat, «L'incendie de l'école de bombardement à Penrhos», Peuples et Frontières, no $3,1^{\text {er }}$ avril 1937, p. 51-54.

53. C. LAINÉ, «Lettre du 18 août 1938», Breiz Atao, n 309, 21 août 1938, p. 4 : «Ar c'hentan Breizad a nac'has komz gallek ouz barnerien an estren"».

54. C. LAINÉ, «Discours de C. Lainé», Breiz Atao, n 310, 4 septembre 1938, p. 3. 55. Allbrogat, «L'incendie de l'école de bombardement à Penrhos», art. cit.

56. U.R., «A Gile !», Gwalarn, n ${ }^{80}$, juillet 1935, p. 47. 
«Er vro na anavez

Na kozni na kleñved

Na tra divalo,

Na maro $^{57} . »$
(Au pays qui ne connaît ni vieillesse ni maladie ni vilaine chose, ni mort.)

Lainé se veut également chevalier, héritier des guerriers celtes. Plusieurs poèmes exploitent la veine soldatesque, comme cette octosyllabique "Vérité celtique» créée le 26 mars 1942, laquelle témoigne d'un effort de versification :

«Gwirionez keltiek

Hoc'h enebour a ziskarit

Neuze keneil a gavit

Ha tonkadur a sevenit.

Kasaomp hor gall milliget

Neuze karomp hor c'hileed

Atao meulomp hon tremened

C'hoant hag ober, rez e vezimp En eur c'hoantaat, sklaer e welimp En eur ober, trec'h e vezimp ${ }^{58}$.»
(Vérité celtique

Votre ennemi vous abattez Alors vous trouvez compagnon Et vous réalisez la destinée.

Haïssons notre Français maudit Alors aimons nos comparses Toujours louons nos trépassés.

Désir et action, nous serons loyaux En désirant, nous verrons clairement En faisant, nous serons victorieux.)

Lainé se fait soldat, chef, prophète :

«Ma youl hini eur meuriad eo E dolzenn bouezusañ Me an hini e lak da vont E youl a zo ac'hanon ${ }^{59}$.»
(Ma volonté c'est celle d'une tribu Son éminence principale C'est moi qui la fait aller Je suis sa force.)

Sa poésie, en fait, est le reflet de ses tourments. À ce titre, deux textes méritent d'être confrontés ${ }^{60}$. Le premier, intitulé Nedeleg hag ar soudard, en français «Noël et le soldat», confronte la veillée de Noël d'un intérieur bourgeois, douillet et confortable, à celle d'un

57. U.R., «TIR NA n-OG», Gwalarn, n 80, juillet 1935, p. 45-46.

58. CRBC, Fonds Lainé, CL6 M329 - poèmes et chants, janvier/mars 1942.

59. Dans Kanaouenn den an dud, CL2 M32 - notes, souvenirs, réflexions, janvier 1942.

60. Urien RIWALLON, «Nedeleg hag ar Soudard» et «Pedenn ar Varc'hegourien», Gwalarn, n $^{\circ} 66$, mai 1934, p. 34-38. 
soldat solitaire - le narrateur - que le bonheur du travail accompli réchauffe dans la nuit froide d'un pays étranger. Le soir de Noël permet aussi de mettre en scène une imitation christique :

«Ha d'an eur a c'hanas

An hini a roas e vuhez

Dre garantez,

E vezo gantañ en iliz.

Ha kor an ograou

A gano evitañ nemetken

Trec'h an emroüsted

Hag ar garantez.»
(Et à l'heure qui engendra

Celui qui donna sa vie

Par amour,

Il ${ }^{61}$ sera avec lui dans l'église.

Et le chœur des orgues

Chantera pour lui seulement

La victoire du dévouement

Et de l'amour.)

La thématique guerrière du soldat christique - abondamment réactivée pendant la Grande Guerre - n'est pas surprenante dans les années 30, mais elle permet ici de mettre en évidence, encore en 1934, la foi catholique de Lainé, qui transpire aussi dans le second poème : une «Prière des chevaliers ${ }^{62}$ ». Un chef, soldat lui-même, «porte-parole» (komzer), s'adresse à sa troupe, dont les hommes ont comme lui abandonné famille, biens et honneurs, pour l'avenir de la Bretagne :

«Komzer : $\quad$ Astennet omp war c'horre an douar,

Ni hag hon eus karet hon Nesa,

Bevet, stourmet, ha marvet evitañ.

Mes hor Skouer e ren en Neñv,

Hag hor galvo stok d'e galon,

ha n'hor bo ket ar pep gwella?

Ar re all : $\quad-$ Hor bo ar pep gwella.»

(Porte-parole : Nous sommes face à terre,

Nous qui avons aimé notre Prochain,

Vécu, lutté, jusqu'à mourir pour lui.

61. Il s'agit du soldat.

62. Une traduction partielle de ce poème est proposée dans Francis FAVEREAU, Anthologie de la littérature bretonne au XXe siècle, tome 2, «Breiz Atao et les autres en littérature, 1919/1944», Morlaix, Skol Vreizh, 2003, p. 499. Le second poème est également publié après guerre dans $\mathrm{Al} \mathrm{Liamm,}{ }^{\circ} 41$, novembredécembre 1953, p. 121-122, et simultanément dans Barzhaz, Al Liamm-Tir na N-Og, 1953, p. 121-122. 
Mais notre Exemple règne au Ciel

Et nous appellera auprès de son cœur,

Et n'en aurons-nous pas le meilleur?

Les autres : $\quad-$ En aurons le meilleur (sic).)

Pour autant, ces prières ne doivent pas faire illusion : elles sont le chant du cygne de la foi chrétienne de Lainé, qui se place désormais au cœur de sa propre foi : à la fois célébrant et célébré, il est le prêtre et le prophète d'une Bretagne à venir. C'est bel et bien une cérémonie païenne qui remplace Noël. En fait, c'est à partir de 1934, alors qu'il se fait scientifique et Breton, alors qu'il rencontre Gerhard von Tevenar ${ }^{63}$, proche de Friedriech Hielscher et des mouvements mystiques non-conformistes allemands, que Lainé renonce au catholicisme. C'est avec von Tevenar que Lainé vit sa première cérémonie païenne, en 1935, à Loos-les-Lille ${ }^{64}$. Il mettra quelques mois à devenir Neven Henaff ${ }^{65}$. Cette nouvelle vision du monde, à travers le prisme de la Bretagne et de la langue bretonne, s'est accompagnée d'une nouvelle façon d'être : Lainé, chef militaire et spirituel, s'imagine druide. «Hervez doare-ober an Drouized gwechall, e kemennomp eeun ha piz hor c'helennadurez d'ar re a gav deomp ez int barrek da vezañ kelennet » (Selon les manières des Druides d'antan, nous dispensons directement et consciencieusement notre enseignement à ceux que nous jugeons aptes à être instruits), annonce-t-il pendant des manœuvres à Rochefort-en-Terre en $1942^{66}$. C'est en ce sens qu'il faut comprendre le discours qu'il fait en 1938 à Saint-Aubin-du-Cormier, où il exhorte ses troupes à la victoire ${ }^{67}$, ou encore pendant les périodes d'instruction militaire, quand il fait part

63. Membre fondateur de la Deutsche Gesellschaft für Keltische Studien, ou Société allemande des études celtiques, von Tevenar établit dès les années 30 un lien discret mais important entre les services de l'Abwehr et les minorités européennes.

64. CRBC, Fonds Lainé, CL8 M410 - calendriers de la foi celtique calculés par C. Lainé.

65. Il se fait appeler Neven vers 1936. CRBC, Fonds Lainé, CL1 M15 - fragments autobiographiques.

66. C.L.K., «Penaos ober gant ar wirionez», Gwalarn, n 148-149, mai-juin 1942, p. 276-280.

67. Ab ArZEL, «Ret eo d'eomp trec'hi (Prezegen Ab-Arzel e St-Albin)», Breiz Atao, $\mathrm{n}^{\circ} 302,15$ mai 1938 , p. 1-2. 
à ses soldats de ses visions ${ }^{68}$. En breton, il s'adresse à une minorité choisie, une élite dans laquelle il se reconnaît, ceux qu'il désigne du néologisme de kileed, ses comparses, ou celui de Brezoned, les Bretons conscientisés. Avec eux, il est, comme dans sa poésie, dans le registre de l'intime. Le breton qu'ils utilisent leur permet de se reconnaître. Pour Lainé, cela passe même par une manifestation corporelle.

Le $1^{\text {er }}$ avril 1937, Olier Mordrel met des gants pour présenter, dans la rubrique "Tribune des jeunes» de sa revue Stur, l'article d'un certain Allbrogat ${ }^{69}$. La livraison, intitulée «Nos deux bases : Irlande et Prusse», n'est pas le fait d'un original, prétend Mordrel, qui ne se prive pourtant pas de recadrer parfois le texte avec force annotations. L'article est bien sûr de Lainé, qui cherche à mettre en évidence ce qui incorpore la Bretagne à la voûte nordique dont la base occidentale est l'Irlande et l'orientale la Prusse. Se basant sur Dottin $^{70}$, des linguistes celtisants, César et sa Guerre des Gaules, il dresse un tableau qui lui permet de comparer quelques mots en latin, vieux celtique, irlandais, allemand, gallois, breton, anglais. Il constate que breton et allemand sont les deux seules langues aryennes à avoir évolué de la même manière; finalement, celtisme et germanisme sont «deux aspects différents d'un seul et même nordisme». La manœuvre vise évidemment l'ennemi latin : «Ainsi le mot kant dans daou c'hant et tri c'hant s'est, en ce qui concerne la consonne initiale, tout a fait rapproché de hund-ert; il est aussi loin que celui-ci du latin centum (kentoum), et n'a plus rien de commun avec le français cent (prononcé sañ) et l'espagnol ciento (syento)», n'hésite-t-il pas à écrire. Mais Lainé va bien plus loin, prétendant que la langue, «étiquette nationale qu'on met sur la pensée internationale ${ }^{71} \gg$, témoigne des mentalités des races :

68. Ab ARZEL, «Gweledigez Kadoran», Stur, n 12, janvier-mars 1938, p. $27-$ 32. Une traduction partielle de ce texte est proposée dans Francis FAVEREAU, Anthologie de la littérature bretonne au XXe siècle, op. cit., p. 499-501.

69. Allbrogat, «Nos deux bases : Irlande et Prusse», Stur, n ${ }^{\circ}, 1^{\mathrm{er}}$ avril 1937 , p. 55-65.

70. Henri-Georges DotTin (1863-1928), linguiste et professeur de langue celtique à l'Université de Rennes.

71. CRBC, Fonds Lainé, CL6 M391 - notes linguistiques, non daté. 
«Examinons maintenant la question des labiales. Entre toutes les langues aryennes, et même par rapport au germanique, le celtique a pris dans la Haute-Antiquité, précisément aux temps de sa plus grande extension et quand il a probablement imprimé à la culture nordique son esprit héroïque et aristocratique décisifs, une physionomie tout à fait particulière par la chute du $p$ initial des mots aryens. D'autre part, il ne comportait pas de $f$. Depuis lors, les langues celtiques n'ont pas persévéré dans cette direction.

Je ne veux pas me perdre dans les détails d'une hypothèse que je ne fais d'ailleurs qu'entrevoir. Remarquons seulement qu'en général les lettres $p$ et $f$ sont celles qui produisent la plus grande déformation apparente de la figure de celui qui parle. Ce sont les deux labiales fortes. Les labiales exigent un mouvement des lèvres très apparent sur la figure, et non pas dentales, palatales et gutturales qui ne font que mouvoir que des organes internes à l'abri de la face. D'autre part, $p$ et $f$ étant des consonnes fortes, produisent une plus grande déformation de la face que les labiales faibles $b, m$ et $v$. La conclusion de ceci serait que les anciens Celtes n'aimaient pas, même en parlant, perdre la face, et ceci en suggère long sur leur mentalité telle que nous la connaissons par ailleurs. Qu'avons-nous fait de l'honneur et de l'héroïsme de nos pères? Mais nous avons toujours leur sang dans nos veines, et notre langue porte encore les traces de leurs initiatives dans lesquelles nous n'avons pas persévéré.»

L'argumentaire peut aujourd'hui prêter à rire, mais la note de bas de page qu'Olier Mordrel ajoute à ce texte précise ce qu'il y a derrière le visage impassible du Celte de Lainé :

«Il est un fait que l'irlandais moderne est encore la langue qui provoque le moins de mouvements de la face. (...) D'une manière générale, on parle gaélique presque sans remuer les lèvres ni les mâchoires. (...) Tous les Celtes doivent à leur langue de conserver en parlant une retenue d'expression et une immobilité du visage qui est pour beaucoup dans l'impression de distinction que donnent souvent chez eux les plus humbles paysans. Sachons gré à Allbrogat d'avoir noté le rôle de notre langue dans la discipline de notre comportement extérieur. Laissons les Latins, Orientaux et autres descendants du singe à leurs gesticulations et à leurs grimaces, indices d'un "tempérament" que nous ne leur envions pas. » 
Lorsqu'éclate la guerre, Lainé cesse quasiment de publier. Désormais, son action se concentre sur ses deux grands projets : son armée bretonne, sa foi celtique. Dès 1938 il commence à rédiger des prières, mais c'est au début des années 1940 que son syncrétisme religieux prend forme. Un imprimé, vraisemblablement publié en 1943, et intitulé God ar Gelted ${ }^{72}$ résume sa vision du dieu des Celtes :

«E gentañ neuz eo E liested

E eil - unanded

Edrede - hollegez

God a leugn hag a zalc'h pep ec'honder.

E gentañ neuz eo E gemmusted

E eil - Zalc'husted

Edrede - Beurbadelez

God a leugn hag a zalc'h an holl amzeriou.

E gentañ neuz eo E Hollc'hallusted

E eil - Red untuek

Edrede - Zreistelez

God a leugn hag a zalc'h pep emskiant».

(Son premier aspect est Sa multiplicité

Son deuxième - Son unité

Son troisième - Sa totalité

God remplit et contient tout espace.

Son premier aspect est Sa variabilité

Son deuxième - Sa Permanence

Son troisième - Son Éternité

God remplit et contient tous les temps.

Son premier aspect est Son innombrable Possibilité

Son deuxième - unique Nécessité

Son troisième - Sa Perfection

God remplit et contient toute conscience.)

Cette nouvelle vision du monde s'accompagne logiquement de la création d'un nouveau calendrier, comme cela se pratique au même

72. CRBC, Fonds Lainé, CL8 I25 - God ar Gelted, s.d. 
moment dans les milieux völkisch allemands. S'inspirant de celui de Coligny ${ }^{73}$, Lainé calcule en 1942 un calendrier de la foi celtique, dont la première année correspond à 1934, année de sa rencontre avec von Tevenar ${ }^{74}$.

Cette foi a donc sa théorie, mais aussi sa langue. C'est logiquement en breton que le fidèle s'adresse à son dieu. Ce choix trouve une explication simple, dans un courrier adressé par Alan Heussaff à Lainé en octobre 1944 : les dieux n'aiment pas que l'on s'adresse à eux dans la langue de l'ennemi; seuls des mots d'origine celtique ou de vieux breton traduisent correctement la pensée religieuse du groupe. Ainsi il n'est même pas souhaitable d'imaginer une prière en français et de la traduire ensuite ${ }^{75}$. Le recours à une langue ancienne permettrait ainsi d'accéder au secret des origines, démarche propre aux religions, lesquelles sont une explication du monde. Aussi, les prières mêlent-elles l'amour, la lumière et tout l'or des retables que Lainé a vu dans son enfance, à la guerre, au courage et à la force, au printemps et à la jeunesse, au vent et aux nuages, aux vivants, aux morts :

«Aotrou an Nevez-Amzer, Da c'hervel a reomp!

Ren ac'hanomp, Doue skedus!

Ren ac'hanomp, Aotrou ar sklerijenn!

Bennig ar sap yaouank er c'hoadou hag er pradou!

Bennig al lu yaouank er brezel hag er peoc'h!

Bennig Da re en Neñv ha war an douar ${ }^{76}$.»

(Seigneur du Printemps, nous T'appelons !

Dirige-nous, Dieu étincelant !

Dirige-nous, Seigneur de la lumière !

Bénis le jeune sapin dans les bois et les prés !

73. Lainé a longuement étudié ce calendrier, et a livré son étude dans Célestin LaInÉ-KerJeAn, "Le calendrier celtique», Zeitschrift für keltische Philologie und Volkforschung, Band XXIII, Helfs3, 1943.

74. CRBC, Fonds Lainé, CL8 M410 - calendriers de la foi celtique, calculés par C. Lainé, 1942.

75. CRBC, Fonds Lainé, CL3 C22 - lettre d'Alan Heussaff à Célestin Lainé, 21 octobre 1944.

76. CRBC, Fonds Lainé, CL8 M538 - prières, incantations, chants. 
Bénis la jeune armée dans la guerre et dans la paix ! Bénis les Tiens au Ciel comme sur la terre.)

Ces prières sont récitées, peut-être chantées, notamment lors de la fête de Ginivelez, située à la fin de l'année, à la fois Noël et Saint Sylvestre. Car enfin, cette foi celtique, bien qu'assez obscure, ou justement parce qu'elle l'est, compte quelques fidèles, qui se réunissent à l'occasion de baptêmes ou mariages, dont un est célébré le 14 juin 1941 dans la forêt de Rennes. À cette occasion, Lainé fixe, étape par étape, le déroulement de la cérémonie. Celui-ci est imprimé sur un petit livret cartonné intitulé An euredi keltiek ${ }^{77}$. Au dos du livret, Lainé a précisé :

«Kenta eured bet lidet d'ar 14vet a Vezeven 1941, d'eur sadorn da greisteiz, ouz troad eun dervenn e Koad Roazon. Dibabet e oa bet da aroueziou ar gwaz, eur skourrig iliao hag eur c'hi du "penn-kaz" ha da re ar vaouez, jenoflennou gwenn hag eur vuoc'h wenn. Dibabet e oa bet gant ar priediou eul liamm liou ruz.

Maen an eured a vezo hini ar vadeziant d'an holl vugale bet ganet da heul an eured-se. Kemend-all gant an dour hag an hanaf.

Kantoellou an eured a zo re badeziant ar priediou.»

(Premier mariage célébré le 14 juin 1941, un samedi à midi, au pied d'un chêne dans le bois de Rennes. Avaient été choisis comme symboles de l'homme, une petite branche de lierre et un chien noir "à tête de chat" et pour ceux de la femme, des girofliers blancs et une vache blanche. Les époux avaient choisi un lien de couleur rouge.

La pierre du mariage sera celle du baptême de tous les enfants à naître de ce mariage. De même pour l'eau et le hanap.

Les chandeliers du mariage sont ceux du baptême des époux.)

Effectivement, la liturgie du baptême est, elle aussi, prévue. Le 7 novembre 1941 est baptisé le premier fils d'un de ses lieutenants, selon un déroulement soigneusement élaboré par Lainé, et rédigé en breton $^{78}$. Une formule officielle baptise l'enfant :

77. CRBC, Fonds Lainé, CL8 I28 - An euredi keltiek, 1941.

78. CRBC, Fonds Lainé, CL8 M521 - organisation du baptême de Budog Peresse, 7 novembre 1941. 


\begin{abstract}
«E vamm a redias e c'henel
Lemm e vaen ha ruz e wiskad

Gantañ e vo trec'h ha brezel

BUDOG d'ezañ zo dereat.»

(Sa mère dut le mettre au monde,

Sa pierre est tranchante et son vêtement rouge

La victoire et la guerre seront siennes

Le nom de BUDOG lui convient.)
\end{abstract}

Parallèlement à cette «liturgie druidique» de pure invention, Lainé met sur pied une armée bretonne. Cette armée connaît plusieurs noms et plusieurs époques. La langue bretonne y tient un rôle prépondérant, comme il s'en explique dans une allocution à ses soldats :

«La langue bretonne est éminemment concrète et humblement sérieuse, voire même pesante - langue de cultivateurs, de marins - de militaires et d'éducateurs - de mathématiciens logiques et de philosophes sereins - d'hommes - et il se vérifie que ce sont les hommes qui y tiennent encore après que les femmes sont passées au français. Le français est la langue de la finesse et de la rouerie, de la légèreté spirituelle, de l'art de parler sans s'engager, des beaux discours, de la mode et même du théâtre si l'on y rajoute les gestes. Langue des déclarations - même des déclamations - de la guerre des mots, guerre des femmes et des diplomates - de la guerre des apparences, guerre des artistes - de la guerre des sentiments, guerre des journaux ${ }^{79}$.

Elle convient parfaitement à ce qu'on appelle la politique, dans le vilain sens du mot, cet ensemble de phrases, d'attitudes, de photos jusqu'au ciné et à la radio, de l'épate généralisée gonflant l'enthousiasme et la crainte à grands coups de pompe démocratique ${ }^{80}$.»

On peut s'étonner que Lainé use de la langue de l'ennemi, mais on connaît l'argument qui veut que l'orateur veuille être compris du plus grand nombre, et tous les soldats du Bezen ne parlent pas breton. Pour promouvoir la langue, il l'associe à quelques valeurs simples :

79. $\mathrm{Ou}$ «jumeaux»?

80. CRBC, Fonds Lainé, CL2 M38 - notes, souvenirs et réflexions, 25 septembre 1942. 
virilité, sérieux, honnêteté, rudesse. Le breton est la langue du corps et de l'action, du guerrier. L'apprentissage du breton lui semble donc un «excellent exercice (...) école et témoignage de volonté persévérante jusqu' au succès ». L'étudiant bretonnant est «ce genre d'hommes qui a la plus haute valeur, celui qu'on recherche dans tous les domaines pour les faire réussir. Partout on se disputera de tels hommes qui sont ceux de l'aristocratie (...). Partout où l'on a un sens véritable des valeurs, on se disputera le concours de ceux qui ont appris le breton ${ }^{81} »$. De fait, Lainé chef accorde beaucoup d'importance à son enseignement : «J'attends que tous l'apprennent assez pour que nous puissions peu à peu bretonniser ces cours », dit-il lors d'une journée d'instruction ${ }^{82}$. D'ailleurs, le breton est savamment distillé dans les leçons de cryptographie. «Pistolet mitrailleur», «George Cadoudal» et «Spahi rouge», constituent avec «Bugel koant», «matez sioul», ou encore «Skol Vreizh» un échantillon disparate mais représentatif de clés de cryptage ${ }^{83}$. Dans cette optique, il est très fier de noter dans son journal que la journée d'examens qu'ont passés les soldats le 27 juin 1943 à Landivisiau s'est entièrement déroulée en breton pour la première fois ${ }^{84}$. Plus encore, il s'adresse parfois à eux directement en breton, dans des circulaires militaires officielles. De même, la hiérarchie du Service Spécial est établie en breton. Lainé, comme il le fit pour la géométrie, s'appuie sur le vieux breton, le vieux celtique, le gaulois, pour justifier la création de mots nouveaux servant à nommer les différents grades. Ainsi, un gour (homme/soldat) fait partie d'un groupe, ou Bod commandé par un kentour (de kintwr, ou kintoviros), groupe lui-même intégré dans un détachement - Ker-commandé par un Kerrenour, lequel obéit un à Kadvan (de Katman, ou Katumanos), commandant d'unité (Bezen), ce Bezen étant une unité d'une armée - Lu - commandée par le Tiern ${ }^{85}$. Dans la même veine, cette armée est logiquement commandée en breton. Elle l'est publiquement lors du pèlerinage à Saint-Aubin-du-Cormier en 1938, obéissant aux

81. CRBC, Fonds Lainé, CL6 M391 - notes linguistiques, non daté.

82. CRBC, Fonds Lainé, CL5 M308 - notes pour une allocution aux hommes du KD, 1942.

83. CRBC, Fonds Lainé, CL5 M246 - cours de cryptographie.

84. CRBC, Fonds Lainé, CL1 M2 - journal juin-juillet 1943.

85. CRBC, Fonds Lainé, CL5 T29 - décisions du Service Spécial, 1942-1943. 15 août 1942. 
«Sounn», «A-zehou... kit! » ou autres «Kuit!» ${ }^{86}$ officialisés dans une circulaire ${ }^{87}$. Cette armée bretonne en constitution s'accompagne en outre d'une paperasserie dûment datée, numérotée et tamponnée, aux armes du Penndael al Lu Vrezhon, ou «état-major de l'armée bretonne». Des papiers militaires estampillés «Stad Vrezon ${ }^{88} »$ sont imprimés sur carton rigide.

Au début de l'année 1943, Lainé envisage la création d'un ordre celtique religieux, militaire et fermé, sur un modèle inspiré par von Tevenar ${ }^{89}$. La seconde condition d'admission, après d'inévitables obligations d'ordre physique, est la suivante :

«... être droit et fidèle dans sa conduite - donc être avoir appris (sic) le breton (Trec'h kenta) - le pratiquer dans sa famille, toutes ses constructions, être en tout un exemple pour les autres, dans le domaine celtique».

On se souvient que le Trec'h kenta est un examen de breton établi par Roparz Hemon dans les années 30, dont Lainé, qui l'a obtenu, tire une grande fierté. Là encore, la langue est liée à des valeurs morales jugées capitales dans l'admission à cet ordre druidique. Après tractations avec les autorités allemandes, cet ordre sera en partie réalisé dans le Bezen Perrot. En partie seulement car le breton, la foi celtique et la mystique guerrière que prône Lainé ne sont l'affaire que d'une minorité proche de l'état-major et décriée par le reste de la troupe, qui méprise ceux qu'elle nomme les «celticards». Bien qu'une heure de breton et d'allemand quasi quotidiennes soient inscrites à l'emploi du temps des soldats, y compris lorsque ceuxci ont fui en Allemagne ${ }^{90}$. Il semble que Lainé n'utilise le breton qu'avec les plus proches de son état-major. C'est l'un d'eux - Yann Bourc'his - qui propose aux bretonnants du Bezen des soirées littéraires lors desquelles on lit du Roparz Hemon et des extraits

86. «Garde à vous», «à droite... droite», «Rompez les rangs !».

87. CRBC, Fonds Lainé, CL5 T41 - commandements bretons, notes sur la cartographie, règles concernant l'émission des messages et signaux.

88. État breton.

89. CRBC, Fonds Lainé, CL8 M480 - projets d'un Ordre Celtique, 20 avril 1943.

90. CRBC, Fonds Lainé, CL5 M253 - emplois du temps et consignes 1944. 
du Barzaz Breiz ${ }^{91}$. C'est un autre chef qui rédige son rapport en breton :

«Lu Brezhon

Perrot Gruppe

\author{
Ar c'herrenour Maout \\ da Rener al Lu
}

Renta-kont

Lakaet em eus bac'hañ ar gour Gwinver e-pad 24 eur evit beza nac'het kanañ epad ar c'herzed ha beza respontet ne felle ket d'ezañ kanañ e brezhoneg, e kave gwelloc'h mont d'an toullbac'h, hag e oa a youl evit chom e-barz keit ha ma karjen.

Eur wech en em gavet er c'harter ne felle mui d'ezañ mont en toull-bac'h. Poan hon eus bet evit hel lakaat da vont tre.

Bez e fell d'ezañ rei e zilez evit kuitaat ar Bezen.

$$
\underline{\text { Maout }}^{92} \gg
$$

«Armée Bretonne

Groupe Perrot

Le Chef de détachement Maout au Chef de l'Armée

\title{
Rapport
}

J'ai mis aux arrêts le soldat Gwinver pendant 24 heures pour avoir refusé de chanter pendant les marches et avoir répondu qu'il ne voulait pas chanter en breton, qu'il préférait aller en prison, et qu'il était volontaire pour y rester tant qu'il me plairait.

Une fois arrivé au quartier il ne voulait plus aller en prison. Nous avons eu du mal à l'y faire entrer.

Il veut donner sa démission pour quitter le Bezen.

Maout.»

91. CRBC, Fonds Lainé, CL5 M259 - annonce de Gevel aux bretonnants.

92. Maout est le pseudonyme de Goulven Jacq. CL5 M268 - rapport de Maout à Célestin Lainé, Hohenentringen, le 8 décembre 1944. 
On remarque ici la juxtaposition du breton et de l'allemand; l'utilisation de pseudonymes celtisants. On voit bien aussi que l'autorité n'est pas une chose si évidente que ça à imposer à une troupe déliquescente, ayant perdu en désertion plus de la moitié de ses effectifs, et on voit enfin que, ne serait-ce qu'en chanson, la pratique du breton, langue des «celticards», ne plaît pas à tous. Lainé a peut-être plus de succès auprès des jeunes Allemands du village de Grendelbuch à qui il chante en breton, le soir à la veillée ${ }^{93}$. Devenu lieutenant SS - Untersturmführer à la fin de l'année 44 - c'est lui qui prend des sanctions contre les soldats récalcitrants :

\section{« $\underline{\text { Tud karteriet }}$}

Da heul danevell an SS-Unterscharführer CONVAL ez eo karteriet e-pad 2 zevezh eus ar 24.12 da greistez betek ar 26 da greisteiz :

SS-Sturmann Marrec

SS-Mann Edwin

Evit: bezañ nac'het meur a wech sentiñ, respontet fall d'o c'hentour ha displeget dirak an holl komzoù damantus d'ar Bezen ha d'an SS.

Karteriet betek ma teuyo urz digant an SS-Untersturmführer WILD diwarbenn ur c'hastiz-bac'h en e genver :

SS-Rottenführer Moreau.

Evit : an hevelep abegoù, ha ouzhpenn : bezañ kinniget e zilez.

\section{Henaff \\ SS-Untersturmführer}

Ar C'hentour Konval a vo lakaet da deurel evez outo ${ }^{94}$.»

« Consignés

Suite au récit du SS-Unterscharführer CONVAL sont consignés pendant 2 journées du 24.12 à midi jusqu'au 26 à midi :

SS-Sturmann Marrec

SS-Mann Edwin

93. CRBC, Fonds Lainé, CL1 M3 - journal 1944-1945.

94. CRBC, Fonds Lainé, CL5 T47 - sanction à l'encontre de Marrec, Edwin et Moreau. 
Pour : avoir refusé d'obéir plus d'une fois, mal répondu au Chef de groupe et tenu devant tous des propos défaitistes sur le Bezen et la SS.

Consigné jusqu'à ce que parvienne un ordre du SSUntersturmführer WILD ${ }^{95}$ au sujet d'un emprisonnement concernant :

SS-Rottenführer Moreau.

Pour : les mêmes motifs, et de plus : avoir donné sa démission.

Henaff

SS-Untersturmführer

Le Chef de groupe Konval sera chargé de les surveiller.»

Les relations entre les hommes du Bezen des derniers instants semblent exécrables. La situation allemande, de plus en plus désespérée, l'explique en partie. On peut y ajouter l'attitude de Lainé lui-même, qui s'évertue à créer autour de lui des structures de plus en plus privées, confidentielles, microscopiques, dans lesquelles on s'exprime en breton. En témoigne la création de la «Couronne Rouge» qui tient une première réunion en décembre 1944, et dont le rôle est défini en ces termes :

«Ar Grennenn Ruz»-Rener al Lu brezhon en deus divizet e vefe er gwellañ ti en hor c'herz ur c'hombod graet ahezañ (sic) hini ar Grennenn Ruz. Lec'h perc'hennet ennañ a vezo d'ar re nemetken a zo bet gloazet da vihanañ en emgann renet ouzh enebourien armet hor Breizh broadel. Den ne c'hello e lemel diganto. Degemer a roint e banvezioù ar Grennenn Ruz d'ar re nemetken a blijo dezho-holl. Skeudennoù ar re lazet en emgann a vo miret ivez eno, dindan warez ar re vev, er c'hombod graet anezhañ en hent-se dre ma tere ar Grennenn d'an drec'hourion ha d'ar re aet da Anaon, hag al liv ruz d'ar Ren Emgann.

(Traduction française à ne pas publier) ${ }^{96}$

Le Commandant du Service Spécial a décidé que dans la meilleure maison que nous occupons il y aura une salle dite de la Couronne Rouge. Ceux-là seulement qui ont été au moins blessés en combat contre les ennemis de la Bretagne nationale

95. Lieutenant d'origine alsacienne commandant le Bezen.

96. Dans le texte. 
y auront une place à eux. Personne ne pourra la leur enlever. Ils accueilleront aux banquets de la Couronne Rouge ceux-là seuls qu'il leur plaira d'y admettre à l'unanimité. Les portraits des morts au combat y seront aussi conservés, sous la protection des vivants, dans la salle ainsi nommée parce que la couronne est l'attribut des vainqueurs et de morts et la couleur rouge est celle des combats ${ }^{97} . »$

Pendant les années de guerre, Lainé se coupe progressivement du monde, du mouvement breton, de son armée même, et de ses potentiels lecteurs. J'ai constaté plus haut le peu de publications de Lainé pendant cette période. Quelques faits saillants doivent cependant être soulignés. En 1943, il publie dans Gwalarn un hommage posthume en breton en l'honneur de son ami Gerhard von Tevenar ${ }^{98}$. Le 20 mars 1944, François Debauvais, chef déchu du mouvement breton d'avant guerre, meurt dans une clinique à Colmar. Dans un ultime courrier ${ }^{99}$, il fait Célestin Lainé légataire du titre Breiz Atao. Lainé, en rupture avec le Parti National Breton de Raymond Delaporte, fonde un parti concurrent du même nom, et publie un numéro de Breiz Atao, le $n^{\circ}$ 337, en mai 1944. Le numéro est quasi intégralement rédigé en français, un seul article en breton rend hommage à l'abbé Perrot ${ }^{100}$. Un autre numéro de Breiz Atao était prévu pendant l'été. Entièrement rédigé en français, à l'exception du texte sur la «Couronne rouge» et d'une ligne dédiée à trois soldats morts en opération, la livraison ne put être publiée du fait de l'arrivée des Américains à Rennes et de la fuite de ses rédacteurs pour l'Allemagne ${ }^{101}$. À ce triste palmarès, on peut ajouter une ultime ligne sur Guy Vissault de Coetlogon dont Lainé apprend l'arrestation, autant dire l'exécution prochaine, en avril 1945, dans son journal intime ${ }^{102}$. Vers la fin

97. Versions bretonne et française dans CL5 T54 - Breiz Atao, $\mathrm{n}^{\circ} 338, \mathrm{p} .11$.

98. C. LAINÉ, «Gerhard von Tevenar», Gwalarn, n 158 , mars-avril 1943, p. $117-$ 119.

99. CRBC, Fonds Lainé, CL3 C15 - lettre de François Debauvais à Célestin Lainé, 22 janvier 1944.

100. C. Lainé-Kerjean, «D’an Ao. Perrot», Breiz Atao, n 337, mai 1944, p. 18.

101. Les tribulations de ce numéro "fantôme» sont relatées par Marcel Guieysse dans Olier Mordrel, Breiz Atao. Histoire et actualité du nationalisme breton, Paris, Alain Moreau, 1973, p. 374.

102. CRBC, Fonds Lainé, CL1 M4 - journal avril-octobre 1945. 
de la guerre, Lainé n'utilise plus guère le breton que pour parler à quelques proches, aux morts, et à lui-même, dans sa poésie, ses journaux intimes. Ainsi, dès le premier jour de la mobilisation, il tient un journal, entièrement en breton, puis le détruit ${ }^{103}$; en 1943, alors qu'il fait passer des examens à ses soldats, il tient un autre journal en breton; enfin, d'août 1944 au printemps 1945, c'est encore en breton qu'il consigne son emploi du temps et ses réflexions. Ce qu'il écrit en avril, pendant les derniers jours du Bezen, est très éclairant sur ce qu'est la langue bretonne pour lui :

«Sadorn 7 - (...) Arouez gwelloc'h n'eus ket. Sioul an amzer; pell'zo emeur o c'hortoz an Amerikaned; prest omp. Mont a reont an holl. N'eus urz ebet deomp nemet chom; Wild a lavar din n'en deus urz all ebet, evitañ e-unan koulz hag evidomp. Neuze e kasomp an holl uniformou da V1, hag an holl armou. War a lavar e c'hellfent dont warc 'hoaz pe devez goude ${ }^{104}$. »

(Samedi 7 - (...) Pas le moindre signe d'amélioration. Le temps est calme; il y a longtemps qu'on attend les Américains; nous sommes prêts.

Tous s'en vont. Nous ne recevons aucun ordre, si ce n'est de rester; Wild me dit qu'il n'a pas d'autres ordres, pour lui-même comme pour nous. Nous envoyons alors tous les uniformes au V1, et toutes les armes. À ce qu'il dit ils devraient venir demain ou le jour d'après.)

Puis il reprend son journal, 3 jours plus tard :

«10 - quitté Tub ${ }^{105}$. le soir après cérémonie bougie et cercle des mains.

11 - La forêt de sapins couvrant les hauteurs, noire et peu aimable mais hospitalière et protectrice, elle me rappelle la vue du sommet du Mooskopf, la noire forêt sous le ciel noir d'orage - côté oriental - Elle est le visage de la Germanie, peu aimable et peu aimée de qui ne la connaît pas, mais protectrice. Elle protégera encore une fois Celtes contre Romains ${ }^{106} . »$

103. CRBC, Fonds Lainé, CL2 M30 - notes, souvenirs, réflexions.

104. CRBC, Fonds Lainé, CL1 M3 - journal 1944-1945.

105. Tübingen en Allemagne. Le Bezen s'y trouve depuis la fin de l'année 1944.

106. CRBC, Fonds Lainé, CL1 M4 - journal avril-octobre 1945. 
C'est lorsqu'il rend les armes, que tout est perdu, que Lainé revient au français. Il est clair dorénavant que pour lui, le breton est la langue de la guerre, de la mort, et d'un paganisme qui, il en est conscient ${ }^{107}$, le voue à la damnation. De fait, en 1945, Lainé, misérable vagabond, erre de trains en trains, cherchant de quoi manger et où dormir. Il ne trouve guère mieux qu'un bunker où s'entassent les Allemands de «l'année zéro ». Là, dans la chaleur de la nuit, s'échangent les odeurs, les pleurs, les cauchemars, les maladies, les râles d'ébats douteux. Il nomme cet endroit «l'enfer», «l'enfer tiède», plus exactement. Effectivement, alors que d'autres sont morts, victimes du Bezen, lui mettra la moitié de sa vie à se consumer, avant de finir logiquement en cendres, éparpillées à Saint-Aubin-du-Cormier, 38 ans pendant lesquels il ne va plus écrire en breton, mais écrire sur le breton.

En 1945, le monde de Célestin Lainé s'écroule. Désemparé, il se tourne vers la religion et l'écriture. Au contact de Hielscher, et vraisemblablement aussi de Jünger, il redéfinit sa pensée religieuse. D'ultimes prières témoignent de la difficulté des temps, et du syncrétisme religieux élaboré alors :

«Hon Tad a zo en Neñv/

Hoc'h anv ra vezo meulet

Ho kalloud ra zeuy brasoc' $h$

Ho youl ra vezo sevenet en hor Rouantelezh hag en Neñv penn da benn,

Lakait ac'hanomp da anavout Ho mennadou /

Nemet e vefent dimp re-vras bec'h da zougen./

Roit deomp kalon evit o seveniñ./

Ha na lakait man m'hon eus kammet outo,

(Evelse bezet graet!)»

(Notre Père qui êtes aux Cieux

Que votre nom soit loué

Que votre pouvoir augmente

107. CRBC, Fonds Lainé, CL1 M25 - fragments autobiographiques. 
Que votre volonté s'accomplisse en notre Royaume et aux Cieux intégralement,

Faites-nous savoir vos intentions

À moins qu'elles ne nous soient trop difficiles à assumer.

Donnez-nous du cœur pour les accomplir.

Et ne nous en tenez pas rigueur si nous les faussons,

(Ainsi soit-il !))

Cette prière, inventée en janvier 1946 pour la fête de Ginivelez, célébrée en Allemagne, s'adresse à «un dieu (...) par exemple notre Père, Toutat ${ }^{108} \gg$. Selon Lainé, la religion chrétienne est une imposture, laquelle s'est emparée de rites anciens, celtiques et gaulois. Les références au «Notre Père» catholique, le rythme même des phrases, la conclusion, ne sont donc pas pour lui des emprunts à la liturgie chrétienne, mais un retour à la tradition celtique. Pour autant, cette religion celtique s'exprime de plus en plus rarement en breton. C'est désormais en français que Lainé écrit. Et il écrit énormément. En 1946, il tient simultanément un journal intime, un carnet de théologie, il rédige son autobiographie, écrit une vie de saint Ronan, une autre de saint Tanguy, et une histoire de la submersion de la ville d'Ys. Le breton était la langue de la guerre, pas la sienne en tant que Breton. Il s'en explique, évoquant sa découverte de Tanguy Malmanche :

«(...) j'eus un premier aperçu de ce qui m'apparaît celte en fait de littérature. Je dis celte et non point de langue bretonne. Sans doute je professe que ce qui est celte ne se développe convenablement que dans son milieu naturel, le milieu de la langue celtique, mais je ne puis admettre que les quelques (sic) deux cent mille noirs des États-Unis qui s'expriment en gaélique aient plus de titres à se prétendre celtes que la majorité des Irlandais et Écossais qui l'ont désappris, voire qu'une bonne partie des Anglais, des Français et des Allemands occidentaux ${ }^{109}$.»

Partant de ce principe, Lainé ne publie presque plus en breton. On lui connaît trois articles après guerre : Lukian Raoult lui attribue une étude sur les noms de lieux, parue dans Tir Na N-Og en $1947^{110}$,

108. «Pedenn d'un Doue (d. sk. Hon Tad, Toutat)», CL8 M517 - carnet de théologie, Albersloh, 6 janvier 1946.

109. CRBC, Fonds Lainé, CL1 T2, p. 37 - autobiographie 1946 (enfance - 1931).

110. Neven ABERISPOU, «Dibennou da Stuman anviou-lec'hiou diwar anviou- 
on peut y ajouter deux courts textes dans les météoriques livraisons de Breiz Atao de 1947 et $1949^{111}$. Les articles ultérieurs seront des textes traduits par Alan Heussaff ${ }^{112}$. C'est que même arrivé en Irlande, où il retrouve d'anciens membres du Bezen avec lesquels il cohabite un temps, Lainé ne parle presque plus le breton, au point qu'en 1949 il décide avec ses colocataires de ne parler qu'en breton jusqu'à l'heure du thé ${ }^{113}$, décision qui trahit sa mesure d'urgence pour idiome en voie de disparition. Même les prières de Ginivelez se disent maintenant en anglais ${ }^{114}$. Lainé échange quelques lettres avec d'autres exilés du Bezen, écrit un poème intitulé Ar goc'henn war ar $r o z^{115}$, mais cette étrange et autobiographique nature morte est d'abord rédigée en français, puis laborieusement traduite. Et ce n'est qu'à l'occasion que Lainé dispense quelques leçons de breton. Dans ces cours il développe une pédagogie originale :

«(...) la plupart prononcent le breton «comme si que ce serait du français »; et ne font guère d'effort pour changer. Il est vrai qu' une personne est toujours la même quelque langue qu'elle emploie, et a tendance à transporter les intonations, accents et autres habitudes prononciatives de sa langue habituelle aux autres. Dans toutes les classes de breton que j'ai faites - et pas un petit nombre - j'ai toujours engagé mes élèves à commencer par réussir à prononcer le français avec l'accent et toutes les habitudes de prononciation bretonne-comme un bretonnant $100 \%$-. C'est seulement quand "i z-êtté kàpàpp de pārlé kómm toul' mōnn" que je les avisais de se mettre à l'étude du breton, pour ne pas commencer sur de mauvaises habitudes, - rectification ultérieure lente et difficile.

gwez», Tir Na N-Og, mars-avril 1947, p. 18-20.

111. Non signé, «Breiz Atao!», Breiz Atao, n 339, novembre 1947, p. 5-6, et non signé, «Falskredenn Nn. 1.», Breiz Atao, n 340, novembre 1949, p. 2-5.

112. Neven HeNAFF, «Sevenadur Atlantegat», Al Liamm, n 102, janvier-février 1964, p. 39-48 (traduction bretonne par Alan Heussaff); Neven HeNAFF, «Deiziadur drouizek Stonehenge», Ar Bed Keltiek, n 110, février 1968, p. 3445 (traduction bretonne par Alan Heussaff), ainsi que deux manuscrits inédits : "Ar fals c'heneliezh», traduction par Heussaff de «La fausse genèse », CRBC, Fonds Lainé, CL8 M472-475, et «Qu'est-ce que la Bretagne», par le même en 1965, CL6 M370.

113. CRBC, Fonds Lainé, CL4 M231 - brouillon de lettre de C. Lainé à Morag.

114. CRBC, Fonds Lainé, CL8 M518 - «Prezegenn Ginivelezh», 24 décembre 1949.

115. «La crotte sur la colline»-CL6 M336, 8 mai 1953. 
Toute mon expérience me conduit à insister encore davantage sur ce point. Il me semble même qu'il y aurait profit à rendre cette méthode obligatoire ${ }^{116}$.»

Ce souci pédagogique répond à une évolution alors récente de la langue, condamnée par Lainé, lui-même très sensible aux différences de parlers locaux. Cette évolution est le fait d'une équipe de bretonnants dont il fut autrefois très proche :

«Pour traduire "son frère est venu avec lui" nos Gwalarnistes, et encore plus nos liammistes, traduisent "Deut eo e vreur gantañ" pe "E vreur 'zo deut gantañ" etc... ce qui n'a pas de $\underline{\text { sens }}{ }^{117}$ pour quelqu'un qui parle breton (= Son frère est venu par le moyen de lui, sans doute sur son dos?). Celui-ci ne peut dire que "Deut eo e vreur asamblez (a-gevret) gantañ" et en français il dira "Son frère est venu ensemble que/avec lui", "Il était ensemble avec /que son frère."

Qu'ils s'efforcent à employer le breton - même mal - c'est bien. À force de l'employer, ils ont des chances de se rectifier (mais aussi de s'endurcir dans leurs erreurs, s'ils n'y prennent garde). Toutefois, où cela ne va plus, c'est lorsque ces gens qui ne savent pas encore le breton se mettent à produire et à écrire, à proposer des modèles littéraires. Leur breton qui n'est le plus souvent qu'un français travesti de mots bretons - mais le prestige des professeurs est tel que Tout-le-monde les admire; et comme à l'intérieur de la Maison Tout le monde se passe la main dans le dos, le résultat est magnifique (sic). Pourtant, depuis Malmanche, il n'y a guère eu que Riou à écrire “en breton” des choses de valeur certaine.

Ces gens auraient beaucoup gagné à réussir à parler le français comme les bretons le parlent : "Si que ç'aurait été lui, qu'il dit,...» "Ma vije bet-heñ, emezañ,...". - "Il va avec la route par les mezou" ... "Mont a ra gant an hent dre (a dreuz d') ar maezou" - etc...

Ceci pour la langue écrite.

Pour la langue parlée, c'est encore pis ${ }^{118}$.»

La charge est directe, qui concerne non seulement un mouve-

116. CRBC, Fonds Lainé, CL6 M386 - notes linguistiques, non daté.

117. C'est Lainé qui souligne.

118. RBC, Fonds Lainé, CL6 M386 - notes linguistiques, non daté. 
ment littéraire - Gwalarn - auquel Lainé lui-même a participé, de la façon qu'on a vue, et l'équipe héritière de ce mouvement animant après guerre la revue Al Liamm. À ces gens, Lainé reproche finalement de se contenter d'un maigre vocabulaire, d'un accent déplorable et d'une syntaxe éloignée du breton populaire. De fait, Lainé a dû entendre du breton populaire, ou du français bretonnisé, à Ploudalmézeau, où il passe beaucoup de temps, contrairement à d'autres, bretonnants de Brest ou de Rennes. L'ancien ami Jakez Riou, bien que Gwalarniste, est épargné, et on connaît la vénération de Lainé pour Malmanche. La personne directement visée dans ce texte est évidemment Roparz Hemon, dont on a vu plus haut l'importance dans la formation bretonne de Lainé. Les écrits de Hemon ont marqué Lainé, qui cite à l'occasion des passages d'An Aotrou Bimbochet e Breiz $^{119}$ aux hommes du Bezen, par exemple dans un discours de novembre $1944^{120}$. Mais en fait, Lainé cumule les griefs contre Hemon. Dès 1938, Lainé avait rédigé une suite à Mentoniez. Il s'agissait d' «un traité de toute la géométrie plane en breton - travail considérable et très soigné, qui m'a pris des années, deux fois plus étendu que Mentoniez publié et bien supérieur à tous points de vue, travail qui ne sera jamais refait, réellement unique pour la langue bretonne ${ }^{121} »$, assure Lainé. Ce manuscrit, que son auteur crut avoir perdu d'abord au début de la guerre, fut confié en 1940 à Roparz Hemon. La maison brestoise de ce dernier est bombardée en 1941, le manuscrit est retrouvé intact. Lainé le récupère, l'emporte à Rennes où la police le saisit en 1944, suite à son départ pour l'Allemagne. À ce travail était joint un manuscrit d'optique géométrique, travail «dont la perte pour la langue de nos mères» l'a beaucoup affecté. D'autres textes furent perdus, comme ce travail réalisé avec le grandpère de Ploudalmézeau : «(...) je collectais auprès des vieux pêcheurs les noms de vingt et trente espèces de goémons, dont la plupart ne sont pas dans les dictionnaires. Gwalarn ne publia pas. Cela fut perdu, comme la fin de la "Géométrie plane" ${ }^{122} »$. Le reproche est à peine déguisé : Roparz Hemon, pour qui Lainé, aidé de sa

119. Roparz Hemon, An Aotrou Bimbochet e Breiz, Brest, Gwalarn, 1927.

120. CRBC, Fonds Lainé, CL5 M310 - discours de Célestin Lainé, Oedsbach, 21 novembre 1944.

121. CRBC, Fonds Lainé, CL3 C123 - lettre de Lainé à Marie, août 1953.

122. CRBC, Fonds Lainé, CL1 M19 - journal 1961-1970, p. 179. Lainé a aussi 
grand-mère, fit le recensement linguistique de toutes les maisons de Ploudalmézeau ${ }^{123}$, est finalement responsable de la disparition de ces travaux, ayant omis de les publier. Le ressentiment, et peut-être aussi l'âge avançant, poussent Lainé à reprocher à Hemon d'avoir refusé des textes qui pourtant ont été publiés dans Gwalarn ${ }^{124}$. Mais Lainé va plus loin :

«Sunger 20 février ${ }^{125}$ - J'ai lu les Barzhonegou de R. Hemon. Je suis frappé de ce qui - pour moi - est une minceur de fond. Et quant à l'expression elle est trop savante à mon goût. Quelques vers sont très bien, une page passe encore, mais à la longue ces flons-flons lyriques et savants me mènent au sommeil comme du Lamartine. Cette poésie m'apparaît comme un poulpe qui vous environne et vous couvre sans que l'on puisse y distinguer ni queue ni patte ni tête ni sens. Un peu comme la musique wagnérienne. Que l'on est loin de la simplicité sèche des anciens récits irlandais, de la nudité de Malmanche. Le "terrible tragique" est ici délayé et noyé dans une mer de bercements poétiques et somnifères.

J'ai l'impression qu'il doit en être de même pour la généralité de ceux qui n'ont vécu leurs épreuves que dans la solitude, dans les pantoufles de l'imagination.»

Ce que Lainé reproche à Hemon, c'est son manque d'engagement, le soupçonnant par exemple de n'avoir pas parlé du procès de 1938 - celui où Lainé refuse de parler français - afin de ne pas se compromettre, par des textes «jugés anti-orthodoxes par le public d'abonnés ecclésiastiques ${ }^{126} »$. De plus, le chef des «gens de la langue seulement» ne se serait pas intéressé à la politique, et n'aurait

collecté des chansons et danses en Léon, dont Polig Montjarret n'a jamais voulu entendre parler.

123. $I d$., ce travail fut vraisemblablement effectué dans le cadre de l'enquête sur la pratique du breton lancée par Gwalarn en 1928.

124. CRBC, Fonds Lainé, CL5 T68 - questionnaire adressé à C. Lainé : Roparz Hemon n'aurait pas publié un article intitulé «The raven» et contenant une allocution donnée à Rochefort-en-Terre en 1942 aux soldats du Service Spécial. Cette allocution, portant sur la vérité, l'amitié, l'ennemi, a pourtant été publiée dans C.L.K., «Penaos ober gant ar wirionez», Gwalarn, n 148-149, mai-juin 1942, p. 276-280.

125. CRBC, Fonds Lainé, CL1 M5 - 20 février 1946, en Allemagne.

126. CRBC, Fonds Lainé, CL1 M5, p. 17 - journal sept. 45 - sept. 47 (Allemagne). 
intégré le Kuzul que lorsque l'occupation allemande fut établie et l'entreprise moins risquée ${ }^{127}$. Et encore, lorsqu'il y siège, Hemon lit ostensiblement son journal pendant les discussions ${ }^{128}$. Les « horribles gens du "mouvement linguistique" ${ }^{129}$ » usent trop leur huile de méninge et pas assez le sang de leur cœur, critique encore Lainé, c'est ce qui explique la médiocrité de leur production. Et c'est là qu'est tout le problème : le breton ne trouve grâce aux yeux de Lainé que dans le cadre de l'action, du combat. Ainsi annonce-t-il en 1953 une réforme orthographique :

«Language - Encore un qui propose une réforme orthographique! Le cri de mépris de la religion offensée (l'orthographie orthodoxe), catholique lorsqu'on dit "Encore un qui propose une hérésie !" De fait, l'attitude d'esprit est la même; un mépris basé sur un dogme. C'est l'expression de l'orthodoxie en orthographe, gouvernée par le Pape régnant, actuellement R. Hemon.

Pourtant, mes bons amis - tout change - un changement est d'abord une hérésie, même avant de devenir un dogme approuvé. Ce qui ne change pas est condamné. Vous devriez le savoir. (...)

J'écris pour ceux qui ont le courage d'admettre que tout n'est pas pour le mieux en ce qui concerne l'état intrinsèque de la langue bretonne. Elle est en changement rapide, et elle le fait afin de pouvoir survivre. Elle doit en sortir très simple - comme l'anglais de dessous l'hégémonie française médiévale - et alors elle peut survivre - comme l'anglais - ou alors elle périra comme le gaélique qui n'a pas su ou pu se simplifier (...)

Toutefois je veux rassurer ces Messieurs de l'Église orthodoxe Gwalarnoliammiste : je ne propose aucun changement présent. Ils peuvent respirer.

Ceci est écrit pour l'usage de ceux du futur, s'ils en ont l'occasion et le goût. C'est à eux que ceci s'adresse.

Pas de changement pour le présent : le breton est si malade qu'il lui faut le repos, comme aussi ceux des arbres en hiver. C'est au printemps, quand la pousse va se produire, qu'on taille

127. CRBC, Fonds Lainé, CL2 M135 - notes, souvenirs et réflexions, mai 1973 à janvier 74 .

128. CRBC, Fonds Lainé, CL2 M119 - notes, souvenirs et réflexions, fin 1971-début 1972.

129. CRBC, Fonds Lainé, CL1 115. 
les arbres. C'est dans la croissance commençante qu'il est utile de faire la culture physique, le dressage, l'éducation, l'amélioration. De plus ceci ne peut se faire qu'avec un pouvoir politique par derrière, un pouvoir qui puisse efficacement contraindre les récalcitrants - et également nécessaire à assurer l'avenir du breton, n'en déplaise à ces messieurs purement littéraires à la mode d'aujourd'hui ${ }^{130}$.»

Force est d'abord de constater, jusque dans le titre même de la note, l'attrait de l'anglais sur Lainé qui, en cette année 1953 où il se fait naturaliser irlandais, le pratique quotidiennement. On remarque ensuite la conception organique de la langue, laquelle croît ou dégénère comme une plante. Au breton, il reproche en fait sa complexité, celle-là même qui permettait jadis d'extraire l'élite de la masse, aujourd'hui jugée responsable de sa disparition progressive. Une rupture s'est produite avec la fin de la guerre. Ce qu'il reproche réellement, et à maintes reprises, au breton livresque de Gwalarn, c'est d'être trop français ${ }^{131}$. On a vu plus haut ce que pensait Lainé de la langue française, langue des beaux discours, de la «guerre des femmes», et de la rouerie. On comprend mieux aussi pourquoi Lainé surnomme Hémon " ar soudard hegarat ${ }^{132}$ », le soldat aimable. Hémon est un faux combattant, son breton trop français n'est pas celui d'un guerrier, son breton n'est pas bon. Aussi, Lainé évoque une réforme à venir, pour des temps futurs : un futur dans lequel Hemon, qui ne l'est qu' «actuellement», ne sera plus le souverain pontife de la langue, un futur dans lequel un État breton aura été instauré. La révolution doit d'abord être politique avant d'être linguistique. Ce principe, énoncé dès la fin de la guerre ${ }^{133}$, repris ici, sera à nouveau martelé quelques années plus tard, mais cette fois dans un article rédigé en anglais et traitant de la langue flamande ${ }^{134}$. De fait, Lainé

130. CRBC, Fonds Lainé, CL6 M336 - «Ar goc'henn war ar roz».

131. La critique n'est pas isolée ; pour une autre, voir CRBC, Fonds Lainé, CL1 M19 - cahier 1961, p. 179.

132. CRBC, Fonds Lainé, CL1 M3 - journal 1944-1945, 13 et 16 novembre 1944

133. CRBC, Fonds Lainé, CL2 M44 - notes, souvenirs et réflexions, 1 er janvier 44.

134. Neven HeuAfF (sic), "The Flemish language in Belgium, 1962», Book of the Celtic League, "The celtic nations», 1963, p. 71-75. Traduction anglaise par Alan Heussaff. 
semble ne plus y croire : il annonce mais ne propose pas. Il ne sera pas le tuteur de la langue malade, pas plus que les jeunes qui n'en auront peut-être pas «l'occasion et le goût». Le breton était la langue des vrais guerriers, elle n'a plus cours en temps de paix. Cette note, inédite, lui permet de vider son sac "gwalarnoliammiste», et de croire qu'il dérange encore, voire qu'il suscite le mépris, comme si - à l'instar de ce qu'il vivait dans ses années d'apprentissage ses relations au breton devaient être pénibles et douloureuses. Mais Lainé ne règle pas ses comptes qu'avec Hemon. Son intérêt pour la philologie lui permet d'aboutir à des conclusions bien surprenantes.

Au milieu des années 50, Lainé se souvient que son grand-père se prétendait d'origine danoise ${ }^{135}$, et que von Tevenar, de passage en Bretagne à la fin des années 30 , aurait trouvé au château de Trémazan un profil des plus vikings ${ }^{136}$. Mettant à profit l'étude des noms des Hébrides et de Man qu'il fit à Galway quelques années auparavant, il certifie que «le nom de Brest (avec forme ancienne Broest) n'est pas celtique», il serait d'origine norroise, c'est-à-dire viking ${ }^{137}$. En 1956, une laborieuse «contemplation des cartes» aboutit aux conclusions suivantes :

«Brest:

Ancienne forme connue Bo-rest - Près de Brest, au fond de rivière de Penfel (la rivière du bout du Vallum), le fort du Bouguen (tjs prononcé Bougen, en breton) et, vers l'intérieur $\underline{\text { Bohars; }}$; ceci est Bo-harz, la frontière, la limite extrême du Bo, vers l'intérieur du Leon.

Mais Bougen $=$ Bo-ugen $<$ Bo-uicinn $=$ Bo-Viking !

Et alors Bo-rest $=$ Bo-hrest $=$ Bo-Chrest $=$ Bo-Christ !

Borest sur la rive gauche, était le campement des chrétiens, Bougen celui des païens au fond de la rivière sur la rive droite je crois et ils y avaient leur vallum d'où le nom de la rivière de Penfel.

D'où le nom de la Recouvrance pour le quartier de la rive droite car Recouvrance fut recouvré sur les païens ${ }^{138}$ !»

135. CRBC, Fonds Lainé, CL1 M13 - souvenirs autobiographiques, 1954.

136. CRBC, Fonds Lainé, CL6 T72 - «Les vikings en Armorique, IX et $\mathrm{X}^{\mathrm{e}}$ siècles», manuscrit entamé avant 1954 sous le nom d'«Histoire de Portsall».

137. CRBC, Fonds Lainé, CL6 M401 - note linguistique, non datée.

138. CRBC, Fonds Lainé, CL6 M390 - «Contemplation des cartes», 28 décembre 
La région brestoise serait en fait une ancienne terre scandinave reconquise par les chrétiens. Ici, les Vikings auraient été bretonnisés. L'argumentation, farfelue, constituée d'un bric-à-brac référentiel de cartes, de guerre des Gaules, de mythologie, de souvenirs familiaux, ne doit pas occulter la logique qui transparait peu ou prou dans ces quelques lignes. Lainé imagine un ultime bastion viking à Tremazan. Pour lui, «pendant près de deux siècles (...) les Bretons de l'Armorique de Ploudalmézeau, quoique continuant à habiter leur pays, ont cessé d'être chrétiens ». Pour démontrer cela, il s'arrange avec l'étymologie bretonne, la fait scandinave, ou l'interprète à sa façon, faisant de Plouguin un Ploe-Viking ${ }^{139}$; de Portsall un «port Norvégien» en vieux breton, du Guilligui, «le chien (de garde) des Norvégiens»; de Kersaint, la «ville de la victoire», «aussi bien en Scandinave qu'en breton»; des Molène et Quemenez «des adaptations bretonnes des noms celtiques scandinavisés du type Guernesey ${ }^{140}$. Lainé malmène le breton, après s'en être pris au linguiste qui fut son formateur et ami. Puisque la lutte est finie, il entreprend la destruction de son breton. On comprend mieux, ainsi, que lorsque pris d'une rechute combattante, et alors qu'il n'est plus apte à écrire lui-même en breton, il fasse traduire ses textes par Heussaff, un autre guerrier.

En fait, Lainé délaisse le breton pour d'autres centres d'intérêt : la transmutation, et la philosophie orientale. Dans ce qui peut être situé dans le temps de ses écrits, les trois derniers textes rédigés dans cette langue datent respectivement de 1953, 1962, et de la fin de sa vie. En 1953, on l'a vu, Lainé méditait sur une colline, en 1962, il se souvient d'histoires et dictons entendus, étant jeune, chez ses grandsparents. Il en tire des conclusions étayant sa réflexion sur le Yin et le Yang ${ }^{141}$. Entre 1'enfance et la vieillesse, la maison de Ploudalmézeau et une sagesse à prétention universelle, le breton de Lainé n'est plus qu'un fragile élastique tendu à l'extrême. Cet élastique casse quelques années plus tard. Vers la fin de sa vie, Lainé griffonne sur tout papier qu'il trouve des pensées plus ou moins compréhensibles, des mots sans lien apparent les uns avec les autres. Sur un emballage de papier

1956.

139. CRBC, Fonds Lainé, CL6 M390 - «Contemplation des cartes», op. cit..

140. CRBC, Fonds Lainé, CL6 T72 - «Les vikings en Armorique...», op. cit.

141. CRBC, Fonds Lainé, CL9 M563 - notes yin-yang. 
kraft, il se remémore quelques expressions, un glossaire extrêmement basique dans lequel "Tomm an heol», "Choum peoc'h», côtoient «kikafarç, kikmoc'h, farçbuan, poulout» ou encore «daouzek, pevarzek... ${ }^{142}$. Ce vocabulaire enfantin, de débutant, exprimé dans une orthographe étonnante (le «ç» à la place du «s $\rangle)$, semble montrer qu'au fur et à mesure des années, Lainé a désappris le breton, la lutte est terminée, il peut mourir.

$\mathrm{Au}$ terme de ce parcours chaotique, il apparait assez clairement que Lainé a inscrit la langue bretonne dans un processus de ruptures diverses. Rupture avec sa famille et le schéma social dont elle faisait la promotion, avec la religion catholique dont l'explication du monde ne le satisfaisait plus, avec sa carrière professionnelle du fait de son engagement breton, avec la France donc, et finalement avec tout ce qui constituait une identité dont il ne voulait plus, bien qu'étant la sienne propre. Après guerre, et sur un mode différent, la langue bretonne reste au cœur de ruptures avec les ténors, vénérés ou déchus, du mouvement breton. Le breton aura été pour Lainé un des instruments de l'organisation de son propre chaos.

Roparz Hemon, qui savait sonder au plus profond des militants, avait bien compris que «le Breton conscientisé est semblable à un homme sur le bord de l'abîme. De l'autre côté de l'abîme, il voit briller sous ses yeux un pays magnifique, un pays à sa convenance, un pays où tout est breton en son sein : langue, territoire, habitudes, etc... ${ }^{143} \gg$. L'image est reprise par Mordrel qui se souvient de ses lectures et promenades bretonnes. «Nous penchions au bord d'un précipice dont personne jamais ne nous avait parlé, mais où une inexorable fatalité nous poussait ${ }^{144} . »$ En 1946, alors que tout était perdu, Lainé écrivait :

«Le pessimisme sérieux et soutenu chez Hemon et encore plus chez De Langlais m'apparait comme enfantillage et manque de goût. N'ont-ils donc jamais goûté le bonheur à la coupe de

142. CRBC, Fonds Lainé, CL6 M396 - notes linguistiques, non datées.

143. Gawain, «Skiant Vreiz», Gwalarn, n 135, avril 1941, p. 321. Cité dans Ronan CAlvez, op. cit., p. 33.

144. Olier MORDREL, op. cit., p. 24 et 96. 
la vie, ce bonheur bref, aigu, que l'on cueille en passant sur la corde raide au dessus de l'abîme, ce petit joyau brillant qui vaut d'autant plus qu'il est environné de ténèbres ${ }^{145}$.»

Ne regrettant rien, se rappelant ses lectures de jeunesse, et peutêtre la couverture de ce roman qui l'avait tant marqué - L'île $d u$ solitaire - Lainé lie le destin de la langue bretonne et le sien propre en une seule, terrible et unique aventure. Or, bien que le breton fût d'abord pour lui l'arme d'un combat contre lui-même, Lainé est resté au bord de l'abîme, quand d'autres y sont tombés.

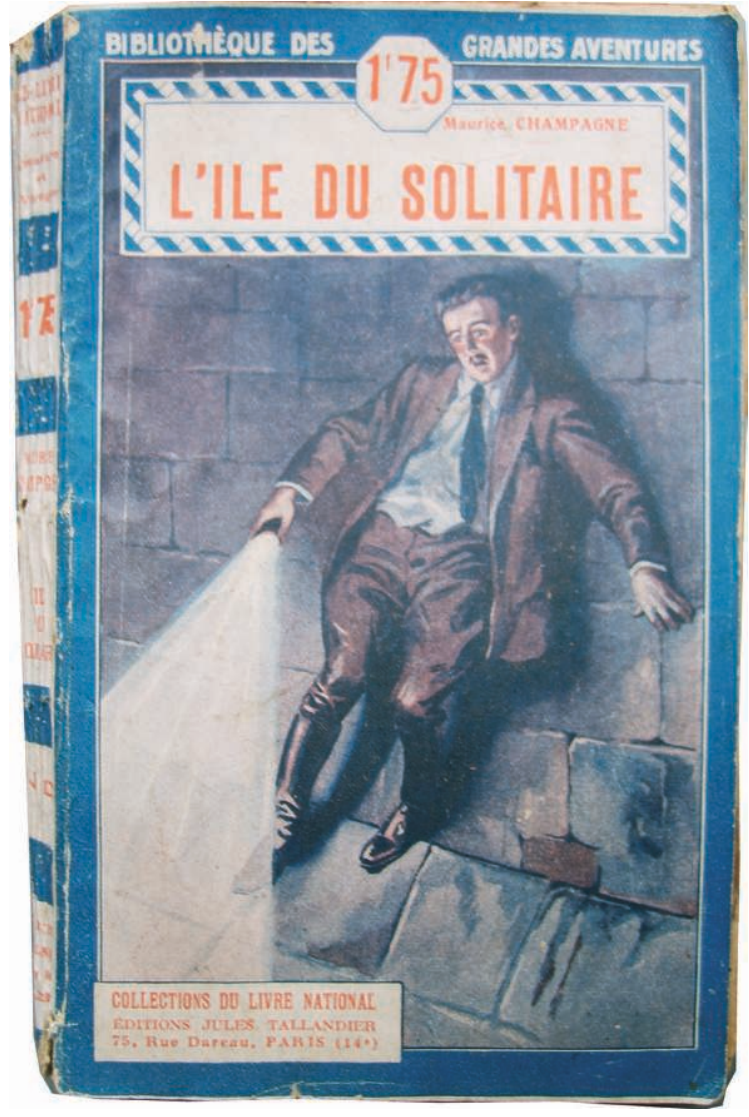

Maurice Champagne, L'île du solitaire, Librairie Delagrave, Paris, 1924

(couverture).

145. CRBC, Fonds Lainé, CL1 M5 - journal sept. 45- sept. 47, 20 février 1946, en Allemagne. 
